\title{
Statistical analysis of vortical structures in turbulent boundary layer over directional grooved surface pattern with spanwise heterogeneity
}

DOI:

$10.1063 / 1.5110048$

\section{Document Version}

Accepted author manuscript

Link to publication record in Manchester Research Explorer

Citation for published version (APA):

Xu, F., Zhong, S., \& Zhang, S. (2019). Statistical analysis of vortical structures in turbulent boundary layer over directional grooved surface pattern with spanwise heterogeneity. Physics of Fluids, 31(8), 085110. [085110]. https://doi.org/10.1063/1.5110048

\section{Published in:}

Physics of Fluids

\section{Citing this paper}

Please note that where the full-text provided on Manchester Research Explorer is the Author Accepted Manuscript or Proof version this may differ from the final Published version. If citing, it is advised that you check and use the publisher's definitive version.

\section{General rights}

Copyright and moral rights for the publications made accessible in the Research Explorer are retained by the authors and/or other copyright owners and it is a condition of accessing publications that users recognise and abide by the legal requirements associated with these rights.

\section{Takedown policy}

If you believe that this document breaches copyright please refer to the University of Manchester's Takedown Procedures [http://man.ac.uk/04Y6Bo] or contact uml.scholarlycommunications@manchester.ac.uk providing relevant details, so we can investigate your claim.

\section{OPEN ACCESS}




\section{Statistical analysis of vortical structures in turbulent boundary layer over directional grooved surface pattern with spanwise heterogeneity}

Fang $\mathrm{Xu},{ }^{1,}$ a) Shan Zhong, ${ }^{1, b)}$ and Shanying Zhang ${ }^{1, c)}$

School of Mechanical, Aerospace and Civil Engineering, University of Manchester,

(Dated: 16 August 2019)

We examine the turbulent boundary layers developing over convergent-divergent riblets (C-D riblets) with three different heights $\left(h^{+}=8,14\right.$ and 20) at $R e_{\theta}=723$ using particle image velocimetry. It is observed that although a logarithmic region presents in the velocity profiles over the converging and diverging line, Townsend's outer-layer similarity hypothesis is invalid. Compared to the smooth-wall case, C-D riblets with a height of $2.4 \%$ of the smooth-wall boundary layer thickness can cause a significant increase in the turbulence production activities over the converging region, as evidenced by a more than $50 \%$ increase in the turbulent shear stress and in the population of prograde and retrograde spanwise vortices. In contrast, the impact of riblets on the diverging region is much smaller. The slope of vortex packets becomes steeper and they are more streamwise stretched in the outer layer over the diverging region, whereas their shape and orientation is less affected over the converging region. Furthermore, the number of uniform momentum zones across the boundary layer increases over the converging region, causing a reduction in the thickness of uniform momentum zones in the outer part of the boundary layer. Overall, while an increased riblet height affects a large portion of the boundary layer away from the wall over the converging region, the impact on the diverging region is largely confined within the near-wall region. Such distinct differences in the response of the boundary layer over the diverging and converging region is attributed to the opposite local secondary flow motion induced by $\mathrm{C}-\mathrm{D}$ riblets.

Cite as: F. Xu, S. Zhong, and S. Zhang, "Statistical analysis of vortical structures in turbulent boundary layer over directional grooved surface pattern with spanwise heterogeneity," Phys. Fluids 31, 085110 (2019).

\footnotetext{
a)Corresponding author; fang.xu@manchester.ac.uk; xu.fluid@foxmail.com

b)shan.zhong@manchester.ac.uk

c)shanying.zhang@manchester.ac.uk
} 


\section{NOMENCLATURE}

\begin{tabular}{|c|c|}
\hline$x(m)$ & streamwise coordinate \\
\hline$y(m)$ & wall-normal coordinate \\
\hline$z(m)$ & spanwise coordinate \\
\hline$u\left(m s^{-1}\right)$ & streamwise velocity \\
\hline$v\left(m s^{-1}\right)$ & wall-normal velocity \\
\hline$U_{\infty}\left(m s^{-1}\right)$ & freestream velocity \\
\hline$U_{\tau}\left(m s^{-1}\right)$ & friction velocity \\
\hline$\Delta U\left(m s^{-1}\right)$ & difference in time-averaged streamwise velocity \\
\hline$v\left(m^{2} s^{-1}\right)$ & kinematic viscosity \\
\hline$\delta(m)$ & $99 \% U_{\infty}$ boundary layer thickness \\
\hline$\theta(m)$ & boundary layer momentum thickness \\
\hline $\operatorname{Re}_{\theta}(1)$ & momentum thickness Reynolds number \\
\hline $\operatorname{Re}_{\tau}(1)$ & friction Reynolds number \\
\hline$K(1)$ & acceleration parameter \\
\hline$\Lambda(m)$ & riblet wavelength \\
\hline$h(m)$ & riblet height \\
\hline$s(m)$ & riblet spacing \\
\hline$\gamma(1)$ & riblet yaw angle \\
\hline$w_{c}(m)$ & riblet crest width \\
\hline$w_{r}(m)$ & riblet root width \\
\hline$A_{g}\left(m^{2}\right)$ & groove cross section \\
\hline$U_{c}\left(m s^{-1}\right)$ & convection velocity \\
\hline$W_{x}(m)$ & streamwise width \\
\hline$H_{y}(m)$ & wall-normal height \\
\hline$\kappa(1)$ & von Kármán constant \\
\hline$B(1)$ & log-law constant \\
\hline$y_{d}(m)$ & zero-plane displacement \\
\hline$S(m)$ & spanwise spacing \\
\hline$\lambda_{c i}\left(s^{-1}\right)$ & swirling strength \\
\hline
\end{tabular}




$\begin{array}{ll}\left\langle\lambda_{c i}\right\rangle\left(s^{-1}\right) & \text { signed swirling strength } \\ \omega_{z}\left(s^{-1}\right) & \text { vorticity in } x-y \text { plane } \\ \Pi(1) & \text { population density of vortices } \\ R(1) & \text { spatial correlation function } \\ \sigma(1) & \text { standard deviation } \\ L_{x}(m) & \text { streamwise length scale } \\ \alpha(1) & \text { inclination angle } \\ N_{U M Z}(1) & \text { number of uniform momentum zones } \\ t_{U M Z}(m) & \text { thickness of uniform momentum zones } \\ |\cdot| & \text { absolute value } \\ \rightarrow & \text { vector } \\ - & \text { ensemble average }\end{array}$

Superscript

$+\quad$ normalization in wall units

, fluctuating component

Subscript

$\begin{array}{ll}\mathrm{s} & \text { smooth wall } \\ \mathrm{p} & \text { prograde vortex } \\ \mathrm{r} & \text { retrograde vortex } \\ \mathrm{rms} & \text { root mean square } \\ \mathrm{ref} & \text { reference point of correlation origin }\end{array}$

Abbreviation

C-D riblets convergent-divergent riblets

CL converging line

DL diverging line

PIV particle image velocimetry

UMZ uniform momentum zone

TNTI turbulent/non-turbulent interface

p.d.f. probability density function 


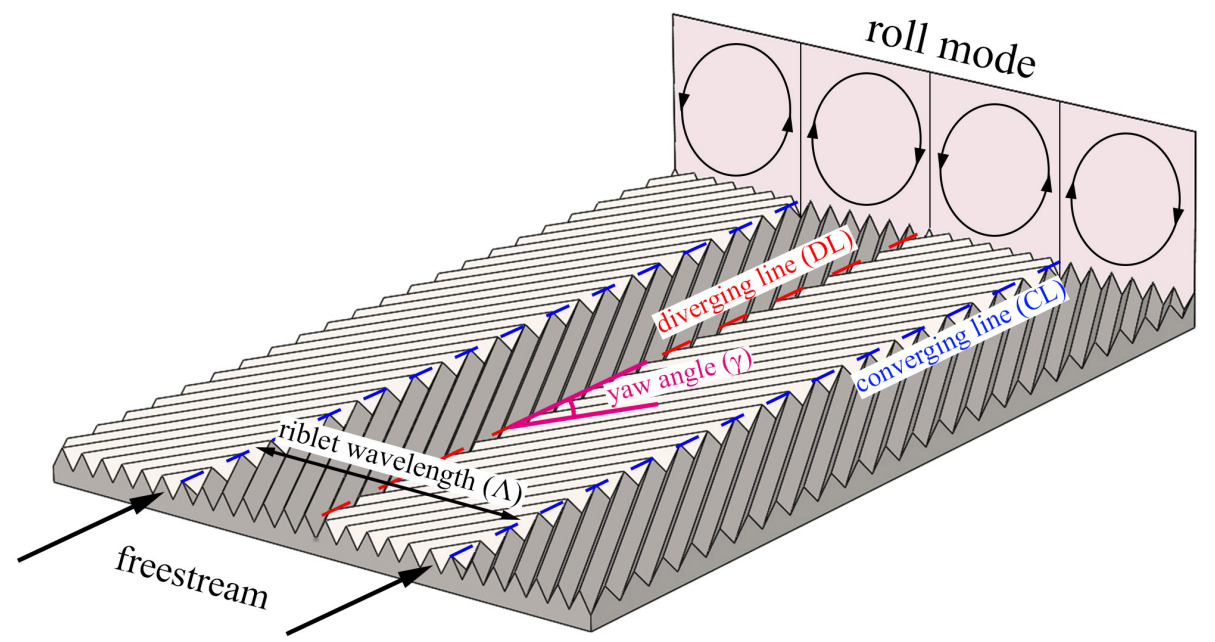

FIG. 1. Schematic diagram of convergent-divergent riblets (C-D riblets) with definitions of diverging line (DL), converging line $(\mathrm{CL})$, riblet wavelength $(\Lambda)$ and yaw angle $(\gamma)$. The time-averaged roll mode is shown in the cross-stream plane.

\section{INTRODUCTION}

Convergent-divergent riblets (C-D riblets) are composed of a spanwise array of sections of ${ }_{10}$ left-yawed and right-yawed microgrooves arranged in an alternating manner, see Fig. 1. Due to their potential for reducing surface friction $\mathrm{drag}^{1}$ and suppressing flow separation, ${ }^{2,3} \mathrm{C}-\mathrm{D}$ riblets have attracted considerable research attentions in the recent few years. ${ }^{4-9}$ Similar to the patterns of spanwise alternating rough/smooth strips ${ }^{10,11}$ or longitudinal elevated/lower surfaces, ${ }^{12-14} \mathrm{C}-\mathrm{D}$ riblets are a type of surface patterns with spanwise heterogeneity, which are capable of generating 15 a secondary flow in the boundary layers. A summary of the published work on C-D riblets can be found in $\mathrm{Xu}$ et al. ${ }^{7}$

As a result of the directional orientation of their microgrooves, C-D riblets generate inherently different flow structures in a boundary layer from those produced by longitudinal riblets. ${ }^{15-21}$ While the effect of longitudinal riblets is known to be confined in the vicinity of the riblet surface, C-D 20 riblets are capable of producing a profound modification to the entire boundary layer. ${ }^{4-7}$ Over the diverging region, the streamwise velocity in the near-wall region is increased compared with that in the smooth-wall case, leading to a reduced boundary layer thickness, and the opposite occurs over the converging region. ${ }^{5,7,8}$ Such a modification to the velocity field has been attributed to the presence of a weak time-averaged secondary flow motion (roll mode) in the cross-stream plane in 25 the boundary layer, whereby an upwelling (downwelling) over the converging (diverging) region 
is created (see Fig. 1). ${ }^{6,7}$ The roll mode is essentially caused by the directional orientation of the yawed microgrooves which results in a surface flow directing from the diverging region to the converging region, as evidenced by the dye visualization experiment conducted by $\mathrm{Xu}$ et al. ${ }^{7}$

The secondary flow motions created by C-D riblets are also found to radically modify both the ${ }_{30}$ time-averaged turbulence characteristics of the boundary layer and the coherent structures. While reduced near-wall turbulence intensity is observed over the diverging region, increased near-wall turbulence intensity is found over the converging region. This is in line with a reduction in the skin friction coefficient over the diverging region and an increase over the converging region as estimated using the modified Clauser technique. ${ }^{5}$ Based on single hot-wire measurements across 35 the boundary layer and PIV measurements in the wall-parallel plane located in the logarithmic region, the roll mode seems to have gathered the low-speed fluid and relocated the very-largescale streamwise structures and features with high vortical activities over the converging region of the C-D riblets. ${ }^{5,6,22,23}$ With a large field of view PIV measurements in all orthogonal planes, Kevin et al..$^{9}$ confirmed that C-D riblets result in formation of large-scale coherent structures over 40 the converging region, which dictate the instantaneous behavior of the roll mode and share some characteristics, such as meandering and breaking, displayed by the superstructures observed in canonical smooth-wall flows.

It is known that a turbulent boundary layer at low Reynolds numbers is populated by horseshoe/hairpin vortices, which are typically composed of either one or two streamwise-oriented legs ${ }_{45}$ connected to a spanwise-oriented head whose rotation is of the same sense as the mean shear. ${ }^{24,25}$ Hairpin vortices are prevalent in the transitional flow and they may not retain a complete hairpin shape in a turbulent boundary layer at a high Reynolds number. ${ }^{26,27}$ In the longitudinal (streamwise wall-normal) plane, the presence of these vortices is manifested by the appearance of 'prograde' spanwise vortices and 'retrograde' spanwise vortices. Prograde vortices, whose rotation is in the ${ }_{50}$ same sense as the mean shear, are often associated with strong ejection and sweep events occurring in the lower upstream and upper downstream regions of their vortex centers, respectively. ${ }^{28}$ Retrograde spanwise vortices, whose rotations are opposite to the mean shear direction, are believed to be the spatial signatures of bent necks of horseshoe/hairpin vortices. ${ }^{29}$ The horseshoe/hairpin vortices are often grouped together in an orderly manner to form vortex packets, resulting in $\delta$-scale ${ }_{55}$ coherent structures which are inclined downstream. ${ }^{24}$ The superstructures, which have a streamwise length scale substantially larger than the local boundary layer thickness, are observed in the outer region, and their motion is a self-sustained process without drawing energy from the hairpin 
vortices in the near-wall region. ${ }^{30,31}$ As such, a hierarchy of vortical structures of different scales are observed in a turbulent boundary layer.

Based on the existing studies, as a type of spanwise heterogeneous surface pattern C-D riblets generate a secondary flow motion due to directional arrangement of their microgrooves. This secondary flow subsequently results in a profound difference in the time-averaged flow field in the converging and diverging region and a redistribution of turbulent activities in the spanwise direction. Therefore, the way the coherent structures organize themselves in the converging and ${ }_{65}$ diverging region is expected to be distinctly different. So far, a fair amount of experimental data has become available enabling a good understanding of the time-averaged behavior of the flow field developing over $\mathrm{C}-\mathrm{D}$ riblets. However, a more detailed description of the activities of coherent structures of different scales is still lacking. Hence further studies are required to gain a new insight in this regard.

Some statistical techniques have been applied to analyze the characteristics of coherent structures in the boundary layer developing along smooth- or rough-wall surfaces. ${ }^{32-34}$ A vortex identification technique was first proposed by $\mathrm{Wu} \&$ Christensen $^{28}$ and Natrajan et al., ${ }^{29}$ to study the population of prograde and retrograde spanwise vortices across a boundary layer. Spatial correlation maps have been widely used to extract the time-averaged shape of hairpin packets. The 75 concept of uniform momentum zones (UMZs) in a turbulent boundary layer was put forwarded by Meinhart \& Adrian ${ }^{35}$ to infer the organized spatial distribution of hairpin vortices. The boundaries of a uniform momentum zone are found to pass through the heads of hairpin vortices, ${ }^{36}$ and hence they are associated with step changes in the instantaneous streamwise velocity profile across the boundary layer. However, these analytical techniques have not been applied to examine the 80 turbulent boundary layer flow developing over C-D riblets or other types of surface patterns with spanwise heterogeneity.

Therefore, in this paper we perform a statistical analysis of the instantaneous velocity vector fields, focusing on the effect of C-D riblets on the characteristics of vortical structures over the diverging and the converging region. Particle image velocimetry (PIV) measurements are con${ }_{85}$ ducted on the turbulent boundary layer over C-D riblets with three different heights. The vortex identification technique is improved in efficiency and applied to study the population of prograde and retrograde spanwise vortices across the boundary layer. Spatial correlation maps are examined to extract the information about the streamwise coherence length and orientation of vortex packets. The number and thickness of uniform momentum zones in the boundary layer are also 
${ }_{90}$ investigated. The effect of riblet height on these properties is summarized.

\section{EXPERIMENTAL SET-UP}

\section{A. Water flume and flat plate}

The present experiment was conducted in a low-speed water flume at the University of Manchester. The water flume has a test section measuring $305 \mathrm{~mm} \times 305 \mathrm{~mm}$ in cross-section. Four metal ${ }_{95}$ screens arranged in a decreasing order of mesh sizes were installed at the inlet of the flume to ensure a low level of background turbulence in the test section. Further details of the water flume can be found in our previous work. ${ }^{7}$

To facilitate the experimental study, a $1350 \mathrm{~mm}$-long flat plate with its test section facing upward was designed, see Fig. 2. It has a super-elliptical leading edge to avoid flow separation from 100 the leading edge. To minimize plate bending along the streamwise direction, the flat plate is strengthened by two angle irons attached to its bottom side. The inclination angle of the flat plate can be finely adjusted using the threads on the four steel legs mounted on the bottom floor of the water flume. There is a small gap between the flat plate and each side wall of the water flume, which restrains the growth of the side wall boundary layers along the flat plate. A cavity measuring ${ }_{105} 500 \mathrm{~mm} \times 250 \mathrm{~mm} \times 6 \mathrm{~mm}$ (in length, width and depth respectively) starting at $550 \mathrm{~mm}$ downstream of the leading edge was machined on the test plate. Rectangular tiles with either a smooth surface or rough pattern as the upper surfaces can be inserted into the cavity of the flat plate.

As shown in Fig. 2, axes $x, y$ and $z$ represent the streamwise, wall-normal and spanwise directions respectively, while $u, v$ and $w$ denote the corresponding velocity components. The ori${ }_{110}$ gin of the coordinate system is located at the beginning of the riblet section, and it levels with the smooth wall and coincides with the center line of the test plate. The instantaneous velocity $\vec{u}=(u, v, w)$ is composed of the time-averaged component $\vec{U}=(U, V, W)$ and the fluctuating component $\overrightarrow{u^{\prime}}=\left(u^{\prime}, v^{\prime}, w^{\prime}\right)$, i.e., $\vec{u}=\vec{U}+\overrightarrow{u^{\prime}}$.

\section{B. Parameters of convergent-divergent riblets}

In the present experiment, C-D riblets were machined on rectangular tiles using 3D (threedimensional) printing technique, as shown in Fig. 3a. The layer thickness and particle diameter were set as $25 \mu m$, and black resin was used to reduce the surface reflection of the laser light. These 


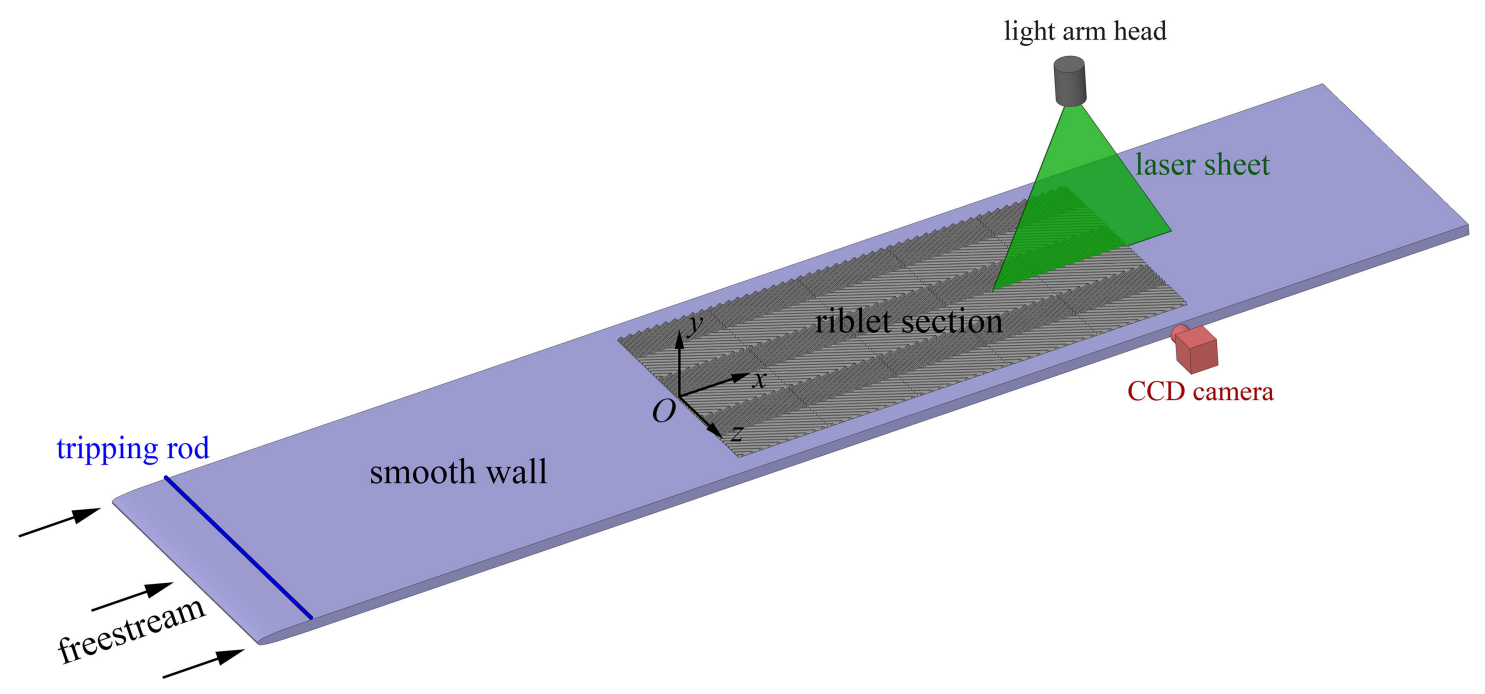

FIG. 2. Schematic diagram of the mono-PIV system in the longitudinal plane. The riblet spacing and the riblet height are not drawn to scale.

riblet tiles can be inserted into the cavity of the flat plate to form the surface pattern. The riblet section is $500 \mathrm{~mm}$ in length and $250 \mathrm{~mm}$ in width. The riblet wavelength $(\Lambda)$ is $83.33 \mathrm{~mm}$, which is ${ }_{120} 2.55$ times of the turbulent boundary layer thickness over the smooth wall $\left(\delta_{s}\right)$ at the measurement station. Across the span of the cavity, three riblet wavelengths were accommodated. The yaw angle $(\gamma)$, which is defined as the angle between the yawed grooves and the freestream direction, is selected as $30^{\circ}$ based on the optimal value proposed by Chen et al. ${ }^{1}$ In our experiment, C-D riblets with three different heights $(h)$, i.e., $0.8 \mathrm{~mm}, 1.4 \mathrm{~mm}$ and $2 \mathrm{~mm}$, and the same riblet spacing $125(s)$ of $2 \mathrm{~mm}$ were tested. In all cases, the smooth wall is leveled with the mid height of the riblets. The blockage ratio, i.e., the riblet height to the boundary layer thickness of the smooth wall $\left(h / \delta_{s}\right)$, is $2.4 \%, 4.3 \%$ and $6.2 \%$ respectively at the measurement station. Over a homogeneous rough-wall surface, the outer-layer similarity is expected to hold for all these three height values $\left(h / \delta_{s}\right){ }^{37,38}$ A flat instead of curved riblet surface was chosen, since the former is easier to machine in future ${ }_{130}$ engineering applications. As indicated in Fig. 3b, to ensure durability, the riblets have trapezoidal teeth with a width of $0.4 \mathrm{~mm}$ at the crest $\left(w_{c}\right)$ and $0.8 \mathrm{~mm}$ at the root $\left(w_{r}\right)$.

The riblet height can be expressed in wall units using the local friction velocity $\left(U_{\tau}\right)$ and the kinematic viscosity $(v)$ as $h^{+}=h U_{\tau} / v$. To ensure consistency, the friction velocity over the smooth wall $\left(U_{\tau s}\right)$ is applied in the normalization. Along the streamwise length of the riblet section, the 135 normalized riblet height $\left(h^{+}\right)$in wall unit varies by less than $6.3 \%$. At the measurement station, the riblet height of $0.8 \mathrm{~mm}, 1.4 \mathrm{~mm}$ and $2.0 \mathrm{~mm}$ corresponds to approximately 8,14 and 20 in wall units respectively, and the riblet spacing in wall units is $s^{+}=s U_{\tau} / v=20$. Hereafter, we refer 
(a)

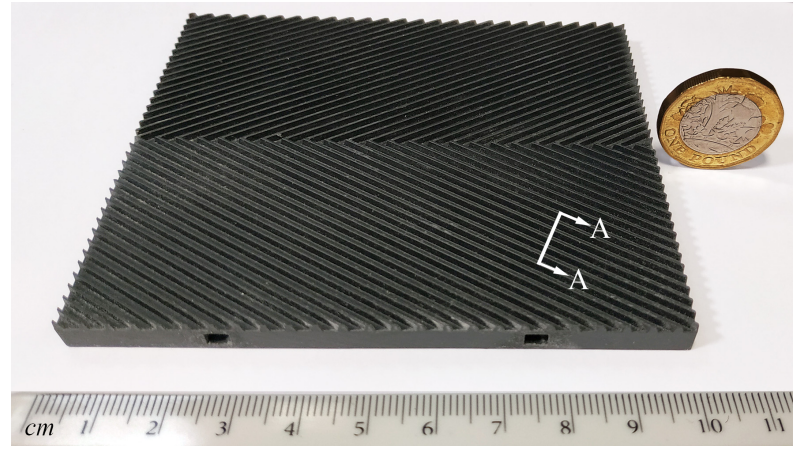

(b)

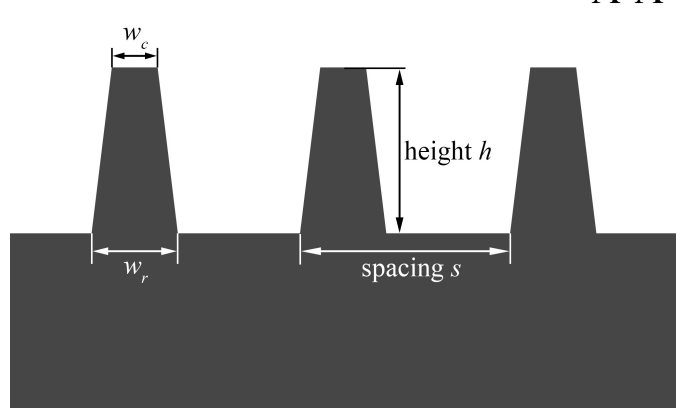

FIG. 3. (a) Perspective view of a tile with C-D riblets on its upper surface. A $£ 1$ coin on its side is displayed for visual comparison. (b) Sectional view of C-D riblets along $A-A$, showing the trapezoidal profile of riblet teeth.

to these C-D riblets as $h^{+}=8, h^{+}=14$ and $h^{+}=20$ riblets respectively. García-Mayoral \& Jiménez ${ }^{39}$ proposed to use the groove cross section $\left(A_{g}\right)$ to characterize the drag reduction effect 140 of longitudinal riblets. The values of $A_{g}^{+^{1 / 2}}=\sqrt{A_{g} v^{2} / U_{\tau}^{2}}$ for the trapezoidal riblets tested in our experiments are 10.6, 14 and 16.7 respectively. Unlike the $h^{+}=8$ or $h^{+}=14$ riblets which stay within the viscous sublayer and the buffer layer, the riblets with $h^{+}=20$ approach the lower bound of the log-law region (see Fig. 4).

Note that the present study and the work by Nugroho et al. ${ }^{5}$ investigate the effect of riblet 145 height from two complementary aspects. In our study, the freestream velocity was kept the same (the same baseline turbulent boundary layer), and the change in $h^{+}$was obtained by changing the physical height $(h)$ of C-D riblets. Since the riblet spacing remained the same, the ratio $h / s$ varied from 0.4 to 1.0. By contrast, in the work by Nugroho et al., ${ }^{5}$ the height of riblets was kept constant, and the riblet height in wall units $\left(h^{+}\right)$was changed by varying the freestream velocity.

150 In their study, the baseline turbulent boundary layers were different, whilst the ratio $h / s$ remained constant. ${ }^{5}$

\section{Particle image velocimetry setup}

The mono-particle image velocimetry (PIV) system used in our experiment is composed of a light-guiding arm, a New Wave Solo-PIV 120 laser generator, a laser pulse synchronizer (Model 155 610036), and a 4-mega pixel charge-coupled device (CCD) camera with 12-bit intensity. The maximum image-capturing frequency of the PIV system is $7.5 \mathrm{~Hz}$. The resolution of the CCD camera is $2352 \times 1768$ pixels. The hollow glass seeding particles have a mean diameter of $10 \mu m$ 
and a density of $1.1 \times 10^{3} \mathrm{~kg} / \mathrm{m}^{3}$, which were estimated to have a settling velocity of approximately $5 \times 10^{-6} \mathrm{~m} / \mathrm{s}$.

Mono-PIV measurements were performed in the longitudinal $(x-y)$ plane, located in the middle of the spanwise direction. The longitudinal measurement plane covers the region located between $12 \delta_{s}$ and $15 \delta_{s}$ downstream of the start of the riblet section, where $\delta_{s}$ is the boundary layer thickness over the smooth wall at $x=450 \mathrm{~mm}\left(\delta_{s}=32.5 \mathrm{~mm}\right)$. The aperture of the lens was set at $f / 4.0$. The time interval between two laser pulses was set at $2 \mathrm{~ms}$. The width of the laser light sheet is around $1 \mathrm{~mm}$. A total of 6000 image pairs (snapshots) were captured to ensure statistical convergence. A low sampling frequency of $0.5 \mathrm{~Hz}$, which corresponds to 12.3 times the turnover time scale in the smooth-wall boundary layer $\left(\delta_{s} / U_{\infty}\right)$, was used to minimize the statistical dependence between adjacent snapshots.

The PIV image pairs were processed using the cross-correlation algorithm in the InSight $4 \mathrm{G}^{\mathrm{TM}}$ 170 software. The first interrogation area is $64 \times 32$ pixels, and the final interrogation area is $24 \times 24$ pixels with a $50 \%$ overlap. The field of view is around $100 \mathrm{~mm} \times 75 \mathrm{~mm}$, which corresponds to a spatial resolution of $0.51 \mathrm{~mm} \times 0.51 \mathrm{~mm}$, or approximately $5 \times 5$ in wall units. The streamwise length of the PIV field is approximately 1000 in wall units. The average particle image diameter is $2-3$ pixels, which ensures the sub-pixel accuracy. The image deformation algorithm is applied 175 considering the high disparity in the displacements of particles at different wall-normal distances. The measurement uncertainties in velocity components are estimated using the Peak Ratio (PR) method incorporated in the InSight $4 \mathrm{G}^{\mathrm{TM}}$ software. The expanded uncertainty in velocity components, i.e., $95.4 \%$ possibility of having the true value within the uncertainty bound, is estimated to be $0.47 \% U_{\infty}$ for the instantaneous flow field and $0.03 \% U_{\infty}$ for the time-averaged flow field.

\section{${ }_{180}$ D. Characterization of the turbulent boundary layer over the smooth wall}

The baseline turbulent boundary layer was tripped by a $4 \mathrm{~mm}$ diameter rod installed $75 \mathrm{~mm}$ downstream of the leading edge of the flat plate. The inclination angle of the flat plate was adjusted to zero degree to minimize the pressure gradient in the freestream direction. The freestream velocity $\left(U_{\infty}\right)$ is $0.2 \mathrm{~m} / \mathrm{s}$. The turbulence intensity in the freestream region is around $0.7 \%$, which 185 is acceptable considering the low Reynolds number of the flow. The acceleration parameter $\left(K=v\left(d U_{\infty} / d x\right) / U_{\infty}^{2}\right)$ is approximately $5 \times 10^{-8}$ in the measurement section, which ensures a streamwise quasi zero-pressure-gradient condition. At the measurement station of $x=450 \mathrm{~mm}$, 


\begin{tabular}{ccccccc}
\hline \hline Parameter & $U_{\infty}$ & $K$ & $\delta_{s}$ & $U_{\tau s}$ & $\operatorname{Re}_{\theta}$ & $\operatorname{Re}_{\tau}$ \\
\hline Value & $0.2 \mathrm{~m} / \mathrm{s}$ & $5 \times 10^{-8}$ & $32.5 \mathrm{~mm}$ & $9.75 \times 10^{-3} \mathrm{~m} / \mathrm{s}$ & 723 & 320 \\
\hline \hline
\end{tabular}

TABLE I. Experimental parameters of the turbulent boundary layer.

the boundary layer thickness $\left(\delta_{s}\right)$ is $32.5 \mathrm{~mm}$ and the friction velocity $\left(U_{\tau s}\right)$ is $9.75 \times 10^{-3} \mathrm{~m} / \mathrm{s}$. The momentum thickness Reynolds number $\left(\operatorname{Re}_{\theta}=U_{\infty} \theta / v\right)$ is 723 and the friction Reynolds number ${ }_{190}\left(\operatorname{Re}_{\tau}=U_{\tau} \delta / v\right)$ is 320. Table I lists experimental parameters of the baseline turbulent boundary layer.

Figure 4 shows the time-averaged streamwise velocity profile $\left(U^{+}\right)$of the smooth-wall turbulent boundary layer. For clarity of presentation, not all the data points in the high $y^{+}$region are presented. The friction velocity $U_{\tau}$ is estimated using the Clauser method with $\kappa=0.4$ and ${ }_{195} B=5.0 .^{40}$ There exist a number of limitations in the Clauser method, including the selection of $\kappa$ and $B$ values and the subjectivity involved in selecting the data points in the logarithmic region. ${ }^{41}$ The profiles of wind tunnel datasets at $R e_{\theta}=617$ (Erm et al. ${ }^{42}$ ) and at $R e_{\theta}=697\left(\right.$ Erm et al. $\left.{ }^{43}\right)$ are included for comparison. DNS datasets at $R e_{\theta}=670\left(\right.$ Spalart $\left.^{44}\right)$ and at $R e_{\theta}=800(\mathrm{Wu} \&$ Moin $^{45}$ ) are also used as references. It can be seen that the measured mean velocity profile ob200 tained from our experiment follows the log-law in the region of $30<y^{+}<120$. While there is a good consistency between our data and the references in the inner layer, some differences exist among these datasets in the wake region of $y^{+}>300$. Besides the minor difference in the Reynolds number, some other factors, e.g., differences in the numerical method used to introduce turbulence, numerical discretization scheme and resolution in simulations or the tripping device 205 used to trigger laminar-turbulent transition in the experiments, ${ }^{43}$ may also contribute to the discrepancies observed. Overall, the baseline streamwise velocity profile is in good agreement with the reference profiles.

Figure 5 compares our profiles of turbulence intensities of $u_{r m s}^{\prime+}$ and $v_{r m s}^{\prime+}(r m s$ refers to the root mean square) with four reference datasets at similar Reynolds numbers. The outer coordinate is 210 applied here to be consistent with reference datasets. Overall, the profiles of turbulence intensities in the present experiment exhibit a very similar trend as the reference profiles. The peak value and its vertical position are similar among these datasets. In the experiments of Erm et al., ${ }^{42,43}$ the lower near-wall peak of $u_{r m s}^{++}$could be attributed to the large value of the hot-wire etched length. ${ }^{46}$ Interestingly, the present turbulence intensity profiles are closer to the published experimental 


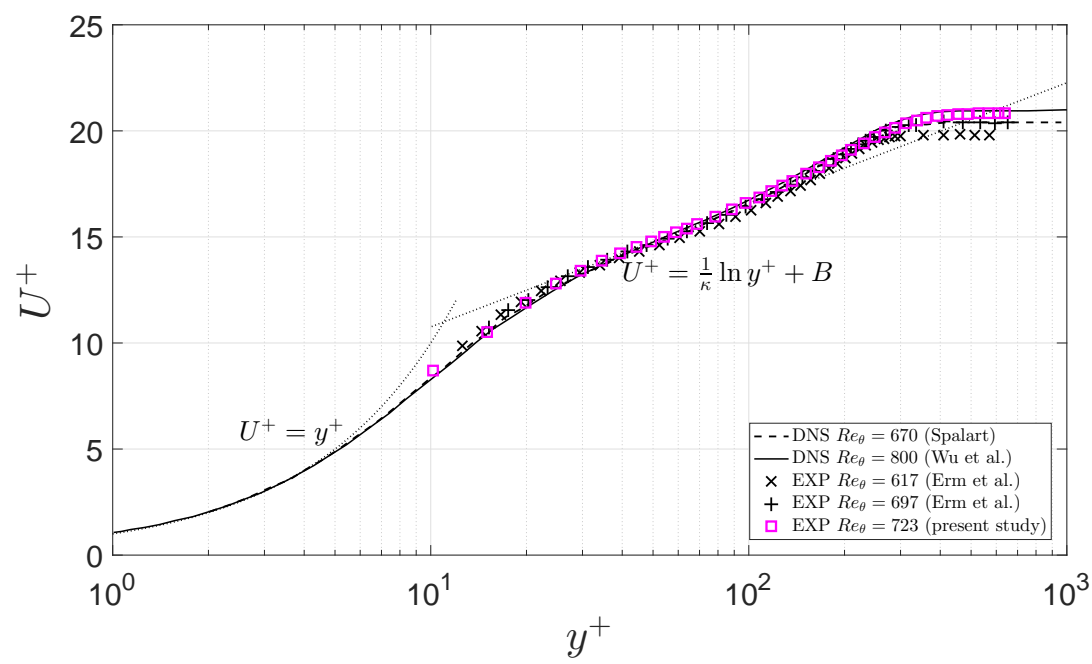

FIG. 4. Time-averaged streamwise velocity profile $U^{+}$as a function of inner coordinate $y^{+}$of the turbulent boundary layer.

(a)

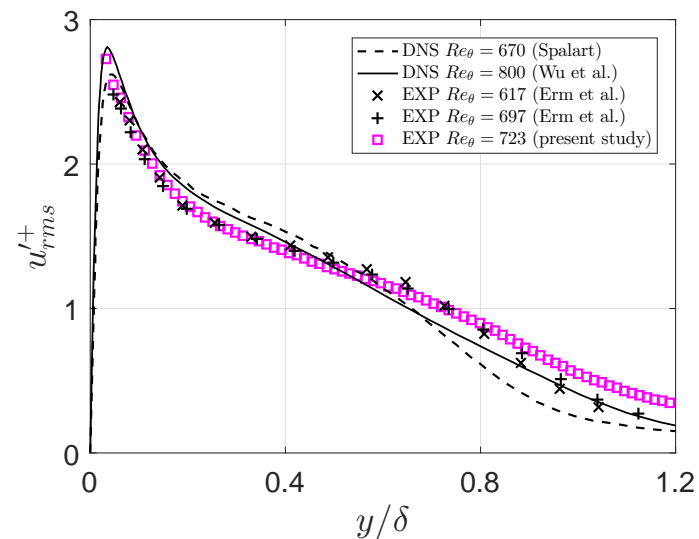

(b)

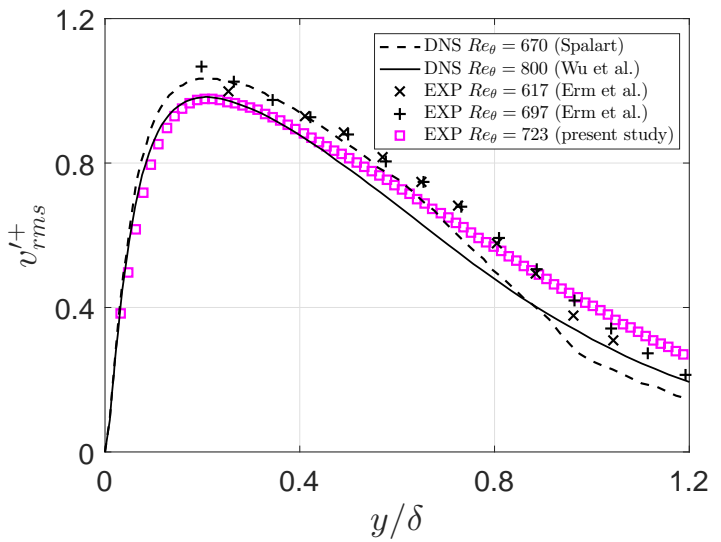

FIG. 5. Turbulence intensity of $(a)$ streamwise component $u_{r m s}^{\prime+},(b)$ wall-normal component $v_{r m s}^{\prime+}$, as a function of outer coordinate $y / \delta$ of the turbulent boundary layer.

${ }_{215}$ data $^{42,43}$ than the numerical simulation results. ${ }^{44,45}$ In the region outside the boundary layer, turbulence intensities of $u_{r m s}^{\prime+}$ and $v_{r m s}^{\prime+}$ in the present experiment are slightly larger than those in the other studies. We attribute this discrepancy to the slightly higher level of background freestream turbulence in our water flume.

Figure 6 compares profiles of the Reynolds shear stress $\left(-\overline{u^{\prime} v^{\prime}}\right)$ of the turbulent boundary layer 220 with four reference datasets. ${ }^{42-45}$ Despite the general agreement with these reference datasets in the profile shape, the peak value of $-{\overline{u^{\prime} v^{\prime}}}^{+}$is slightly lower in the present experiment. At a low Reynolds number, Erm et al. ${ }^{43}$ revealed that the Reynolds shear stress profile is sensitive to the way the tripping device is installed. Thus, the minor variation of $-{\overline{u^{\prime} v^{\prime}}}^{+}$may be attributed to the 


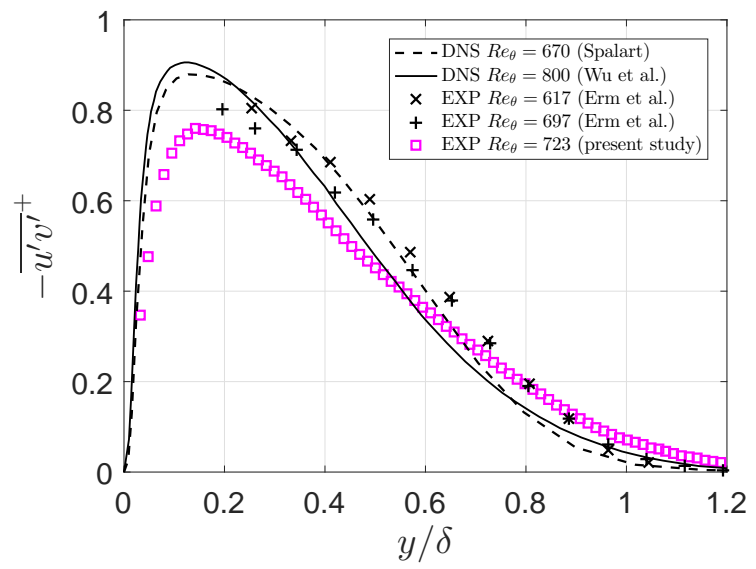

FIG. 6. Reynolds shear stress $-{\overline{u^{\prime} v^{\prime}}}^{+}$as a function of outer coordinate $y / \delta$ of the turbulent boundary layer. size and location of the tripping device used.

\section{III. STATISTICAL TECHNIQUES}

In this section, the statistical techniques used for extracting the characteristics of vortical structures are outlined. To identify spanwise vortices from PIV data, an efficient vortex recognition algorithm based on the queue data structure is utilized, which enables the population density of prograde and retrograde vortices to be computed. The spatial correlation of instantaneous velocity 230 fields is applied to reveal the streamwise extent and inclination angle of vortex packets. The analytical technique to extract the features of uniform momentum zones in a turbulent boundary layer is also introduced.

\section{A. Recognition and distribution statistics of spanwise vortices}

Prograde and retrograde vortices are spanwise vortices which possess negative and positive 235 values of the spanwise fluctuating vorticity, respectively. The signed swirling strength $\left(\left\langle\lambda_{c i}\right\rangle\right)$, as proposed by Zhou et al., ${ }^{47}$ can be applied to identify the instantaneous spanwise vortices by extracting local swirling motion from the mean shear flow. In the longitudinal plane, the definition of the signed swirling strength is given by

$$
\left\langle\lambda_{c i}\right\rangle=\lambda_{c i} \frac{\omega_{z}}{\left|\omega_{z}\right|},
$$

where $\lambda_{c i}$ is the imaginary part of the complex conjugate eigenvalue of the in-plane velocity gra-

240 dient tensor, $\omega_{z}$ is the in-plane vorticity, and the operator $|\cdot|$ denotes the absolute value. The sign 


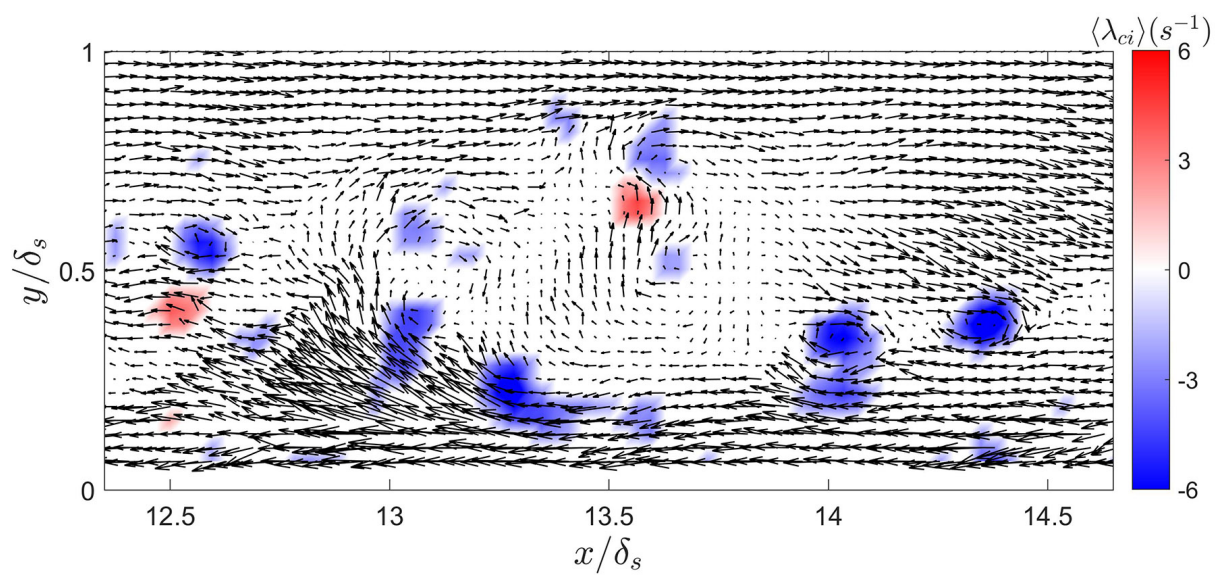

FIG. 7. Sample contour of the instantaneous signed swirling strength $\left\langle\lambda_{c i}\right\rangle$ and in-plane velocity vectors $\left(u-U_{c}, v\right)$ revealing the distribution of prograde (blue) and retrograde (red) vortices.

of the in-plane vorticity $\left(\omega_{z} /\left|\omega_{z}\right|\right)$ indicates the rotational direction of the local swirling motion.

Figure 7 shows the distribution of spanwise vortices in a randomly selected instantaneous field obtained from the present study over the smooth wall. Due to the direction of the mean shear, there are more prograde vortices than retrograde vortices. The in-plane velocity vectors in Fig. 7 are ob245 tained using Galilean decomposition by subtracting a convection velocity of $U_{c}=0.85 U_{\infty}$. The vortical structures which have the same convection velocity can be identified by the closed streamline patterns, and the positions of these structures are aligned with the areas of high magnitude of $\left\langle\lambda_{c i}\right\rangle$.

The regions in the longitudinal plane constituting individual prograde/retrograde vortices can 250 be identified by specifying predetermined thresholds of value and size. The vortex identification technique was first proposed by $\mathrm{Wu} \& \mathrm{Christensen}^{28}$ and Natrajan et al. ${ }^{29}$ In order to further improve computational efficiency and reduce memory usage, a (breadth-first) vortex recognition algorithm based on a queue data structure is proposed. Compared with the brute force method, this algorithm improves efficiency and reduces computer memory usage by using dynamic data 255 structures. ${ }^{48,49}$ Details of this algorithm are listed below.

S1. Scan each point in the instantaneous field to mark the points which exceed the threshold value(s) in terms of the signed swirling strength.

S2. Find a marked point in the field (e.g., the point with the largest $\left\langle\lambda_{c i}\right\rangle$ ), then enqueue (i.e., insert the point item at the back of the queue) and unmark it.

${ }_{260} \mathrm{~S} 3$. While the queue is not empty, dequeue (i.e., remove) the first point. Then enqueue and 
unmark its adjacent marked points (four candidates are the points at the top, bottom, left and right). Repeat this operation until the queue is empty.

S4. The points dequeued in S3 constitute a connected area. Determine whether or not that area can be considered as a prograde/retrograde vortex by evaluating its spatial range.

${ }_{265} \mathrm{~S} 5$. Repeat S2-S4 until there is no marked point left in the field.

To extract the centers of spanwise vortices found with the vortex recognition algorithm, the weighted average of the points constituting a prograde/retrograde vortex is calculated, with the local swirling strength $\lambda_{c i}$ as the weighting coefficient. At a given wall-normal station, the threshold value of the vortex intensity is selected to be proportional to the root-mean-square of the local 270 signed swirling strength, which accounts for the non-homogeneity of the flow in the wall-normal direction. ${ }^{28,50}$ In the following analysis, the threshold of the vortex intensity is selected as 1.5 times of the root-mean-square of $\left\langle\lambda_{c i}(y)\right\rangle$ over the smooth-wall case at the same location. In accordance to the choice of 22 wall units (three grid points) in $\mathrm{Wu} \&$ Christensen, ${ }^{28}$ the threshold of vortex size is set as four grid points in the present study, which corresponds to approximately 20 wall 275 units over the smooth wall. By using the same thresholds obtained from the smooth-wall case, we can quantitatively compare the population of prograde/retrograde vortices over C-D riblets with different riblet heights.

In our study, the population trends of prograde and retrograde vortices are presented as a function of the wall-normal distance. The population density of prograde (retrograde) vortices is de280 fined as

$$
\Pi_{p(r)}=\frac{N_{p(r)}}{H_{y} \cdot W_{x} / \delta_{s}^{2}},
$$

where $N_{p(r)}(y)$ is the ensemble-averaged number of prograde (retrograde) vortices whose centers are located in a rectangular area of $H_{y} \times W_{x} . H_{y}$ is the wall-normal height which is selected as $0.1 \delta_{s}$ (which equals 33 in wall units), and $W_{x}$ is the streamwise width of the field of view of the PIV images. The center of the rectangular area is located at a wall-normal station of $y$. The definition of 285 population density in Eq. (2) is similar to the one proposed by Wu \& Christensen ${ }^{28}$ Thus, $\Pi_{p(r)}(y)$ indicates the normalized population of prograde (retrograde) vortices within an area of $\delta_{s} \times \delta_{s}$ in the longitudinal plane. 


\section{B. Extraction of spatial correlation features}

The vortex packet (hairpin packet), which is composed of a hierarchy of hairpin vortices ar290 ranged in an organized manner, can be analyzed using spatial correlations of the instantaneous velocity fields in the longitudinal plane. The two-dimensional spatial correlation function of streamwise fluctuating velocity fields is defined as

$$
R_{u^{\prime} u^{\prime}}\left(\Delta x, y ; y_{r e f}\right)=\frac{\overline{u^{\prime}\left(x, y_{r e f}\right) u^{\prime}(x+\Delta x, y)}}{\sigma_{u^{\prime}\left(y_{r e f}\right)} \sigma_{u^{\prime}(y)}},
$$

where $\Delta x$ is the streamwise separation, $y_{r e f}$ is the wall-normal distance at which the correlation is calculated, $\sigma$ is the standard deviation, and the overline denotes an ensemble average of all PIV 295 snapshots.

To quantitatively analyze the features of vortex packets, the streamwise length and the inclination angle can be extracted from the spatial correlation contour. With a reference to Christensen $\& \mathrm{Wu},{ }^{32}$ the streamwise length scale $\left(L_{x}\right)$ can be defined as the streamwise distance between the upstream location and the downstream location of the $R_{u^{\prime} u^{\prime}}=0.5$ contour line at $y_{r e f}$.

300 The inclination angle $(\alpha)$ is obtained by best fitting the $R_{u^{\prime} u^{\prime}}$ contour of a specific value with an ellipse which is centered at the location where the correlation is taken. The angle of the major axis of the ellipse forms with the streamwise direction is taken as the inclination angle. ${ }^{51}$ In the present study, the overall inclination angel of the vortex packet is estimated by averaging the inclination angles of the $R_{u^{\prime} u^{\prime}}=0.4,0.5$ and 0.6 contours. The method of finding the inclination angle using 305 the contour lines of intermediate values of correlation coefficients described above is found to yield more robust results than using the contour lines of low values of correlation coefficients. This is because this method is less susceptible to the noise in the correlation field. Christensen $\& \mathrm{Wu}^{32}$ reported that the inclination angle $\alpha$ of the $R_{u^{\prime} u^{\prime}}$ contour gives a good indication of the inclination angle of the vortex packet. The inclination angle is largely invariant over a wide range of Reynolds 310 numbers. ${ }^{52}$

\section{Identification of uniform momentum zones}

The presence of uniform momentum zones (UMZs) in the turbulent boundary layer was first revealed by Meinhart \& Adrian ${ }^{35}$ and further investigated by Adrian et al., ${ }^{36}$ Kim \& Adrian, ${ }^{53}$ de Silva et al. ${ }^{34,54}$ etc. Within such a zone there is a relatively small momentum variation, and 
315 a significant momentum change only occurs across the boundary between adjacent zones. The UMZs are prevalent across a turbulent boundary layer, and they can persist for long distances along the streamwise direction. ${ }^{35}$

Figure 8a illustrates the UMZs in a randomly selected instantaneous field obtained from the present study over the smooth wall. The solid black lines delimit UMZs, and the solid red line indi${ }_{320}$ cates the interface between the turbulent regime and the non-turbulent regime. The region between solid lines has an approximately uniform streamwise momentum. The turbulent/non-turbulent interface (TNTI) is identified using the kinematic energy criterion in the frame of reference moving with the freestream, as proposed by de Silva et al. ${ }^{54}$

Figure $8 \mathrm{~b}$ compares the instantaneous and time-averaged streamwise velocity profiles along the ${ }_{325}$ vertical dotted line in Fig. 8a. There exists a significant change in the instantaneous streamwise velocity across the boundary of UMZs. The horizontal dotted lines delineate the boundaries of UMZs, while the upper dotted line indicates the TNTI. In Fig. 8c, the probability distribution function (p.d.f.) of the instantaneous streamwise velocity is applied to demarcate UMZs. The vertical dotted lines indicate the velocities which delimit UMZs, while the closed pentagrams ${ }_{330}$ indicate modal velocities. The entire field of view of the PIV snapshot in Fig. 8a is accounted to make the probability distribution as smooth as possible. PIV data points in the non-turbulent region are excluded to avoid their dominance in p.d.f. statistics. In the histogram, the value at each peak point (modal velocity) delineates the characteristic velocity within a UMZ, whereas the value at each valley point corresponds to the boundary between adjacent UMZs.

In the present study, the entire PIV field of view which corresponds to $L^{+} \approx 1000$, is taken into account to analyzed features of UMZs, and the non-turbulent regime above the TNTI is excluded. Our parameter study using all PIV snapshots reveals that, when $L>\delta_{s}$ (i.e., $L^{+}>320$ ), the value of $L$ has a negligible effect on the statistical characteristics of UMZs. This result is consistent with the finding of de Silva et al., ${ }^{34}$ which indicates that a selection of $L^{+}$greater than 300 does not 340 affect the p.d.f. of $N_{U M Z}$.

\section{RESULTS AND ANALYSIS}

In this section, after briefly introducing the profiles of velocity and Reynolds shear stress, we reveal the effect of C-D riblets on the vortical structures in three inter-related aspects. The population densities of prograde/retrograde spanwise vortices are compared over C-D riblets and the 
(a)

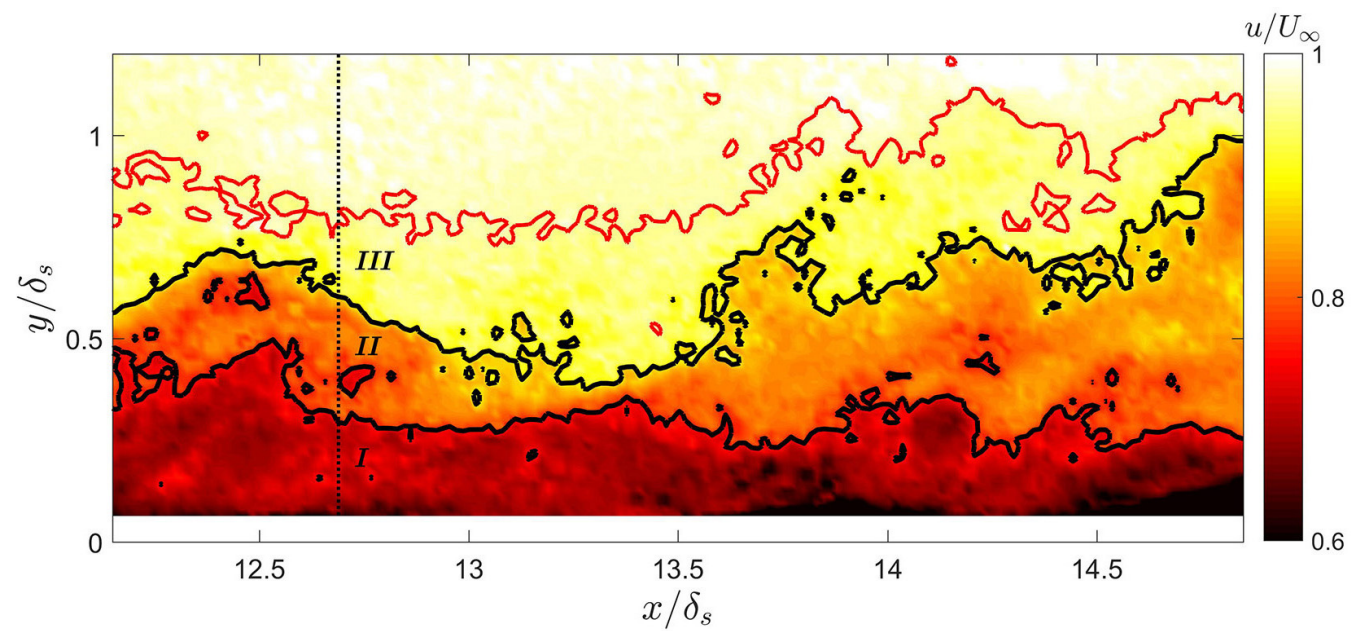

(b)

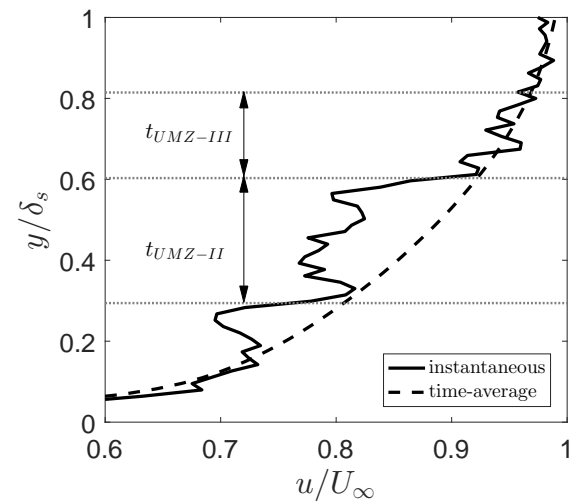

(c)

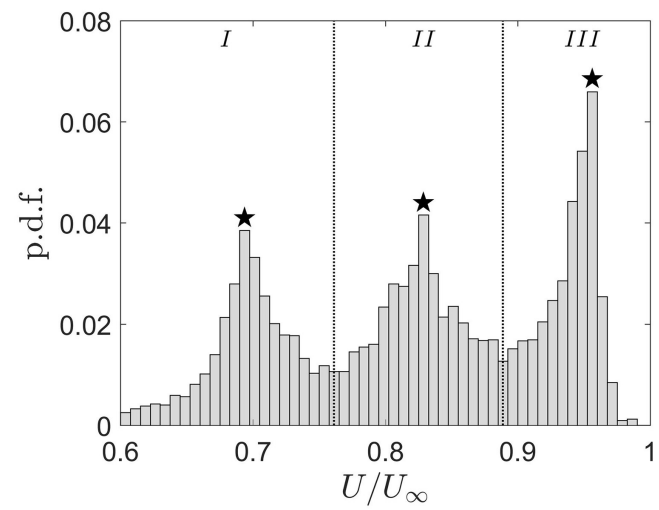

FIG. 8. (a) Sample contour of the instantaneous streamwise velocity $u$ revealing the presence of uniform momentum zones. (b) Profiles of the instantaneous ( $\_$) and time-averaged ( - - - ) streamwise velocity along the vertical dotted line. (c) P.d.f. of the instantaneous streamwise velocity normalized by $U_{\infty}$.

${ }_{345}$ smooth wall. The features of vortex packets, including the streamwise extent and the inclination angle, are extracted and compared. The effect of the newly created vortical structures on the uniform momentum zones is also analyzed. The riblet height effect on these aspects is revealed by comparing three sets of C-D riblets with different heights.

\section{A. Velocity and Reynolds shear stress}

The effect of C-D riblets is first revealed by the time-averaged streamwise velocity profiles over the CL and the DL in Fig. 9a. The converging line (CL) and the diverging line (DL) are defined by the C-D riblets geometry relative to the crossflow, see Fig. 1. Compared to the smooth-wall case, the local streamwise velocity decreases over the CL and increases over the DL. Over the CL, a decrease in the local streamwise velocity is observed in the entire boundary layer. By contrast, 
355 over the DL, the increase in the local streamwise velocity is only significant in the near-wall region. Figure $9 \mathrm{~b}$ shows the profiles in the time-averaged streamwise velocity difference $\left(\Delta U=U-U_{s}\right)$ between $\mathrm{C}-\mathrm{D}$ riblets and the smooth wall at the same wall distance. Over the CL, the magnitude of $\Delta U$ is larger (more negative) as the C-D riblets become higher. Over the DL, an increase in the riblet height primarily affects the near-wall region of $y / \delta_{s}<0.1$. At a higher position of $y / \delta_{s}>0.1$, 360 the magnitude of $\Delta U$ is only slightly beyond zero. The difference between the affected regions, i.e., the entire flow field over the CL while only the near-wall region over the DL, is consistent with the previous observations in the laminar boundary layer ${ }^{7}$ and the turbulent boundary layer. ${ }^{5,6}$ The mechanism can be attributed to the direction of vertical flow and the influence of wall surface. ${ }^{7}$

Figure 9c compares profiles of the time-averaged wall-normal velocity $(V)$ over the CL and the ${ }_{365} \mathrm{DL}$ of $\mathrm{C}$-D riblets and the smooth wall. Compared with the smooth-wall case, a strong upwelling occurs over the CL and an intense downwelling is seen over the DL in the time-averaged sense. It is found that the downwelling over the DL is more intense than the upwelling over the CL in the near-wall region, which is aligned with the previous observation in the laminar boundary layer. ${ }^{7}$ Over the DL, as C-D riblets become higher, the magnitude of $V$ increases continuously, while 370 the vertical peak position remains essentially unchanged. An increased magnitude of $V$ can be attributed to the increased cross-sectional area of the yawed grooves as the C-D riblets become higher. Over the CL, as the riblet height $h^{+}$increases, the magnitude of $V$ becomes larger, i.e, the upwelling turns more intense. The peak position of $V$ over the CL shifts away from the wall as the riblet height increases, which is attributed to the more intense upwelling over this region.

Figure 9d compares profiles of the Reynolds shear stress $\left(-\overline{u^{\prime} v^{\prime}}\right)$ over the CL and the DL of C-D riblets and the smooth wall. The Reynolds shear stress $-\overline{u^{\prime} v^{\prime}}$ indicates the wall-normal momentum transfer due to turbulent events. ${ }^{55}$ Over the $\mathrm{CL},-\overline{u^{\prime} v^{\prime}}$ increases dramatically compared to that over the smooth wall, with a larger riblet height leading to a higher Reynolds shear stress. In a spanwise-periodic in-phase sinusoidal channel, ejection events are preferentially located near the 380 peaks where there present upwelling secondary flows. ${ }^{56}$ These ejection events serve as the main contributor to the high $-\overline{u^{\prime} v^{\prime}}$ observed at the peaks. With an increased riblet height, the vertical position of the Reynolds shear stress peak over the CL shifts away from the wall. The vertical shift of the peak may be attributed to the increased magnitude of the wall-normal velocity over the CL (see Fig. 9c). Over the DL, a less intense Reynolds shear stress than that over the smooth 385 wall is observed, and the lowest riblet height $h^{+}=8$ (open triangle $\triangle$ ) corresponds to the lowest magnitude of $-\overline{u^{\prime} v^{\prime}}$. Since the region away from the wall has a lower magnitude of Reynolds shear 
(a)

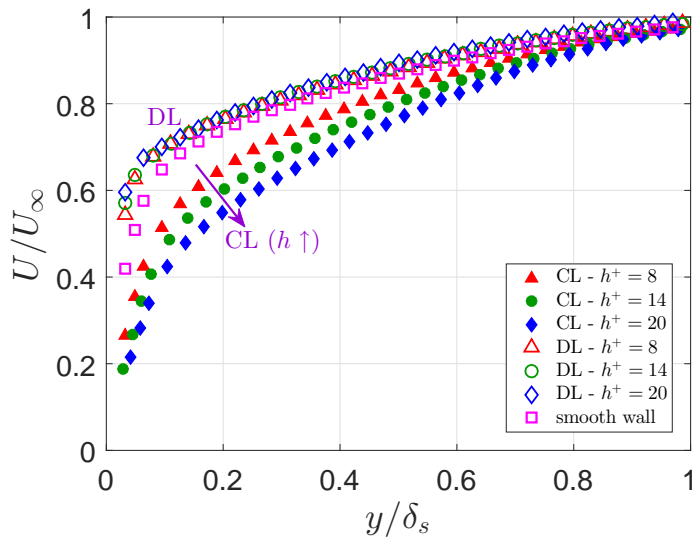

(c)

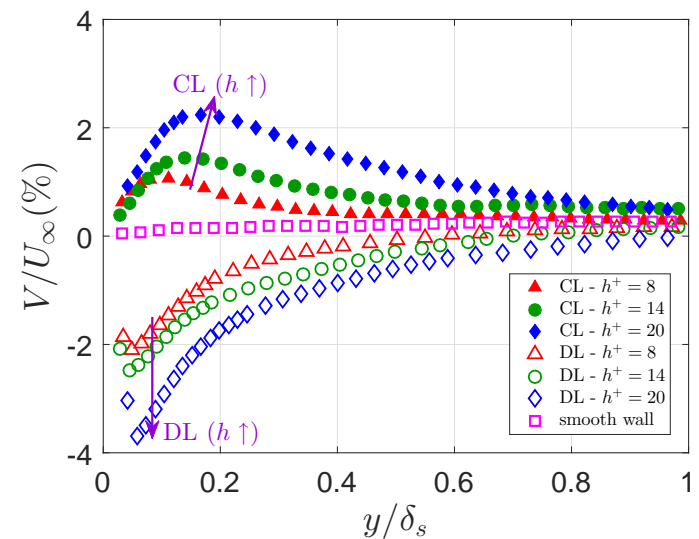

(b)

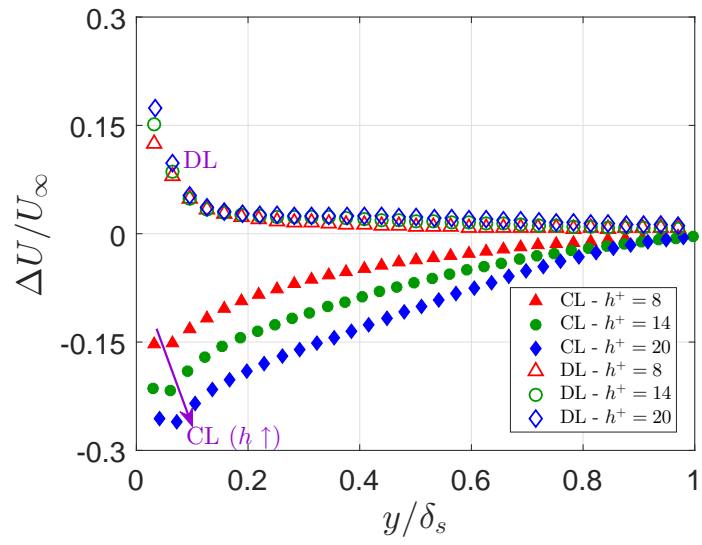

(d)

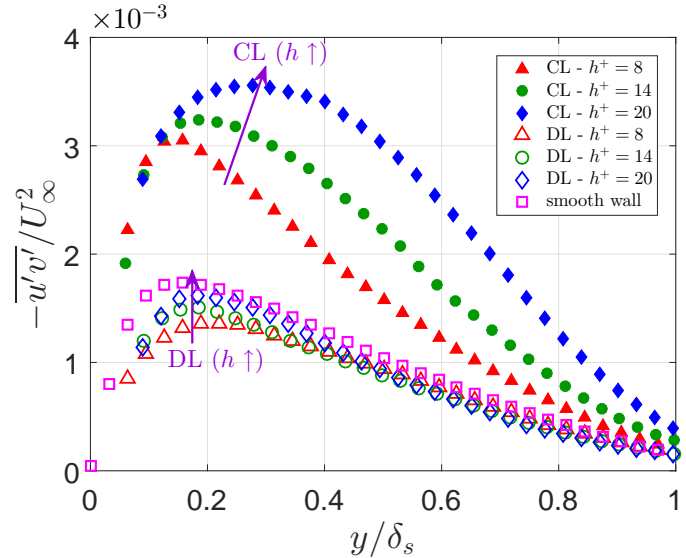

FIG. 9. Profiles of (a) time-averaged streamwise velocity $U,(b)$ time-averaged streamwise velocity difference $U-U_{s},(c)$ time-averaged wall-normal velocity $V,(d)$ the Reynolds shear stress $-\overline{u^{\prime} v^{\prime}}$, as a function of outer coordinate $y / \delta_{s}$.

stress, the downwelling over the DL transfers the fluid with low $-\overline{u^{\prime} v^{\prime}}$ downward, making the local $-\overline{u^{\prime} v^{\prime}}$ smaller than that of the smooth wall. The secondary flow in the turbulent boundary layer becomes more intense as the riblet height increases, which contributes to more fluctuations over 390 the diverging region. Thus, as the riblet height becomes larger, the Reynolds shear stress over the DL slightly increases. A change in the riblet height influences the distribution of $-\overline{u^{\prime} v^{\prime}}$ in the entire boundary layer over the CL and affects only the region of $y / \delta_{s}<0.5$ over the DL.

In summary, C-D riblets lead to an increased local streamwise velocity, a downwelling and a reduced Reynolds shear stress over the DL, and the opposite applies to the CL. The secondary flow 395 becomes more intense as the riblet height increases. For the streamwise velocity and the Reynolds shear stress, the impact of a changing riblet height is mainly reflected in the near-wall area over the DL and in the whole boundary layer over the CL. As the riblet height increases, the crosssectional area of the yawed microgrooves becomes larger, resulting in greater flow capability and 
more intense secondary flow.

\section{B. Wall-shear stress and outer-layer similarity}

To gain an insight on the local wall-shear stress, we analyze further profiles of the streamwise velocity over the CL/DL of C-D riblets and the smooth wall. Figure 10a compares profiles of the time-averaged streamwise velocity, wherein the friction velocity over the smooth wall $\left(U_{\tau s}\right)$ is applied in the normalization. It is clearly seen that the near-wall velocity increases over the DL and decreases over the CL, and a quasi-logarithmic region exists in all velocity profiles over the CL/DL. The slope of the quasi-logarithmic region is larger over the CL and smaller over the DL, and a larger riblet height enhances such a trend. The existence of the log-law region makes it possible to estimate the wall-shear stress using modified Clauser method. Since the time-averaged spanwise velocity is nominally zero over the CL/DL, such analysis could be regarded valid.

As revealed by Clauser, ${ }^{40}$ the surface pattern of uniformly distributed sand grits leads to an increased wall-shear stress and a downward shift of the mean flow in the logarithmic region of the boundary layer. In the modified Clauser method, ${ }^{5,57,58}$ the following equation is applied to the logarithmic law region of the streamwise velocity to estimate the wall-shear stress,

$$
\frac{U}{U_{\tau}}=\frac{1}{\kappa} \ln \frac{\left(y-y_{d}\right) U_{\tau}}{v}+B-\Delta U^{+},
$$

where $y_{d}$ is the zero-plane displacement, $\Delta U^{+}$is the roughness function which represents the 415 vertical shift of the logarithmic region. The friction velocity can be determined by fitting the logarithmic law with three floating variables of $U_{\tau}, y_{d}$ and $\Delta U^{+}$. A positive (negative) $\Delta U^{+}$represents a higher (lower) value of $U_{\tau}$, and thus an increase (decrease) in wall-shear stress. The value of $\Delta U^{+}$ depends on various parameters including the shape and configuration of the surface roughness. To eliminate the term $\Delta U^{+}$, as proposed by Nugroho et al., ${ }^{5}$ Eq. (4) is differentiated with respect to $y$, 420 which leads to

$$
\frac{\Delta U}{\Delta y}=\frac{U_{\tau}}{\kappa} \frac{1}{y-y_{d}} .
$$

The friction velocity $U_{\tau}$, along with the zero-plane displacement $y_{d}$, can be estimated by best fitting the time-averaged streamwise velocity with Eq. (5) in the logarithmic region.

The logarithmic region is manually selected using the linear section in Fig. 10a. Figure 10b shows the same profiles of the time-averaged streamwise velocity presented in Fig. 10a, with the 425 local friction velocity applied in the normalization. The most apparent feature is the downward 


\begin{tabular}{lccc}
\hline \hline$h^{+}$ & 8 & 14 & 20 \\
\hline $\mathrm{CL}$ & $+50 \%$ & $+58 \%$ & $+63 \%$ \\
$\mathrm{DL}$ & $-21 \%$ & $-24 \%$ & $-29 \%$ \\
\hline \hline
\end{tabular}

TABLE II. Variation of wall-shear stress over the CL/DL compared to the smooth wall.

shift of the velocity profile over the CL and the upward shift over the DL, which indicate an increased drag over the CL and a decreased drag over the DL respectively. The estimated changes of the wall-shear stress $\left(\tau_{w}=\rho U_{\tau}^{2}\right)$ are listed in Table II. The changing trend of the local wall-shear stress, i.e., an increase over the CL and a decrease over the DL, is consistent with the previous ${ }_{430}$ estimations. ${ }^{5,6}$ The overall wall-shear stress over C-D riblets is not available in the present experiment. For longitudinal riblets with the same height of $h^{+}=8,14$ and 20 (the same trapezoidal riblet teeth with $s^{+}=20$ ), the variation of the wall-shear stress is estimated to be $-6 \%,-5 \%$ and $-1 \%$, respectively. ${ }^{39}$ Compared to the longitudinal riblets that exhibit spanwise homogeneity, C-D riblets result in a spanwise variation of the wall-shear stress. The spanwise variations in wall-shear 435 stress over C-D riblets may be attributed to the flow topology in the yawed microgrooves observed by dye visualization. ${ }^{7}$ Over the diverging region, the fluid from the upper region is diverted away along the yawed microgrooves rather than directly impinging on the wall surface, resulting in a decrease in wall-shear stress. In contrast, over the converging region, the fluid being transported from both sides collides with each other there, and the wall-shear stress is increased correspond440 ingly. As the riblet height increases, the drag variations compared to the smooth-wall case become more pronounced. With an increased riblet height, the drag increase over the converging region is more significant than the drag reduction over the diverging region. It should be mentioned that, as noted by Nugroho et al., ${ }^{5}$ the estimation accuracy using the modified Clauser method is limited.

Over C-D riblets, the spanwise direction of the secondary flow is from the low wall-shear stress 445 region (diverging region) to the high wall-shear stress region (converging region). Due to the low resistance, the fluid tends to flow in the direction parallel to the yawed microgrooves. ${ }^{7,59,60}$ This contributes to the formation of the secondary flow in order to satisfy the continuity requirement. The spanwise flow induced by the mean flow skewing has a contribution to the large-scale secondary flow over C-D riblets disregard of the state of boundary layer. It should also be mentioned 450 that the turbulence contribution in generating the secondary flow cannot be ignored, especially in the boundary layers at high Reynolds numbers. 
(a)

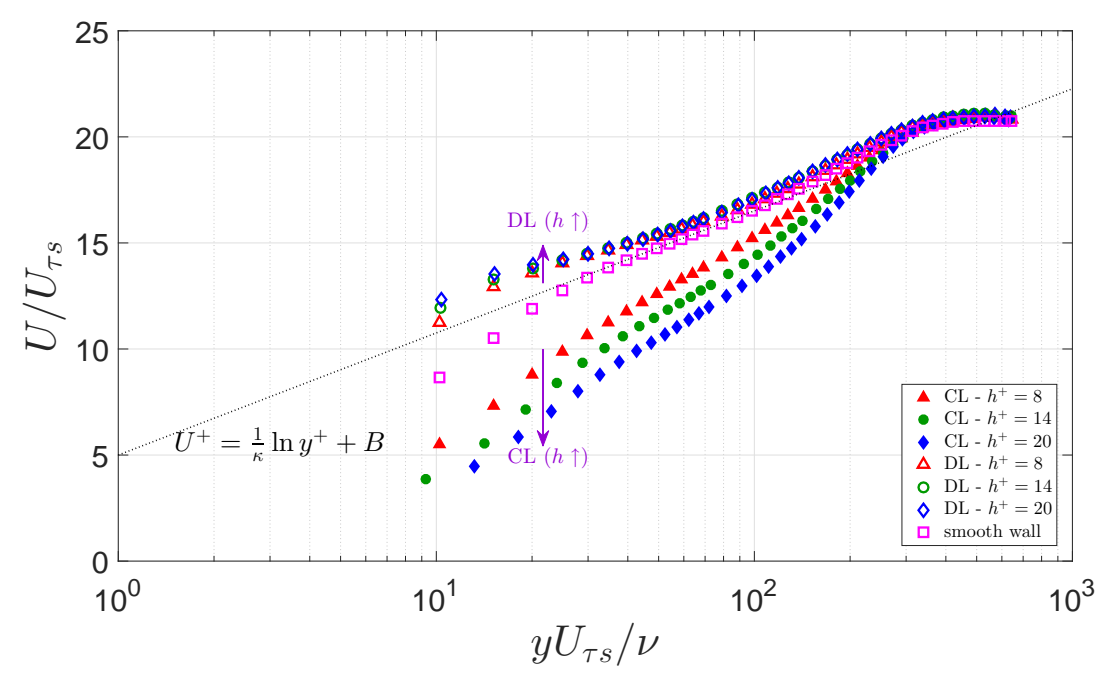

(b)

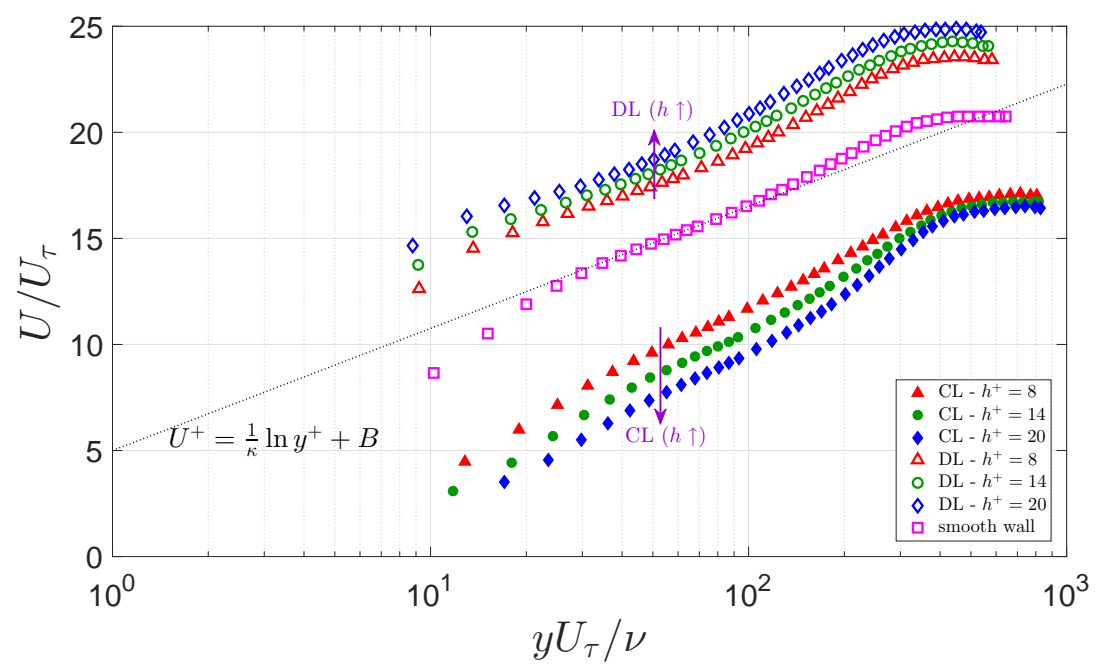

FIG. 10. (a) Time-averaged streamwise velocity profile $U / U_{\tau s}$ as a function of inner coordinate $y U_{\tau s} / v$, where $U_{\tau s}$ is the friction velocity over the smooth wall. (b) Time-averaged streamwise velocity profile $U / U_{\tau}$ as a function of inner coordinate $y U_{\tau} / v$, where $U_{\tau}$ is the local friction velocity.

Townsend's outer-layer similarity hypothesis over C-D riblets, which states

$$
\frac{U_{\infty}-U}{U_{\tau}}=f\left(\frac{y}{\delta}\right)
$$

where $\left(U_{\infty}-U\right) / U_{\tau}$ is the normalized velocity decay, $f(\cdot)$ is assumed universal in the outer layer regardless of the surface pattern. Figure 11 shows the profiles of velocity decay in the outer region 455 over C-D riblets. It can be clearly seen that the velocity profiles do not overlap in the outer region, which indicates that Townsend's outer-layer similarity is not valid over C-D riblets in the present study. The deviation from the baseline (smooth-wall case) is more severe over the CL than that over the DL. This phenomenon can be attributed to the upwelling over the converging region, 


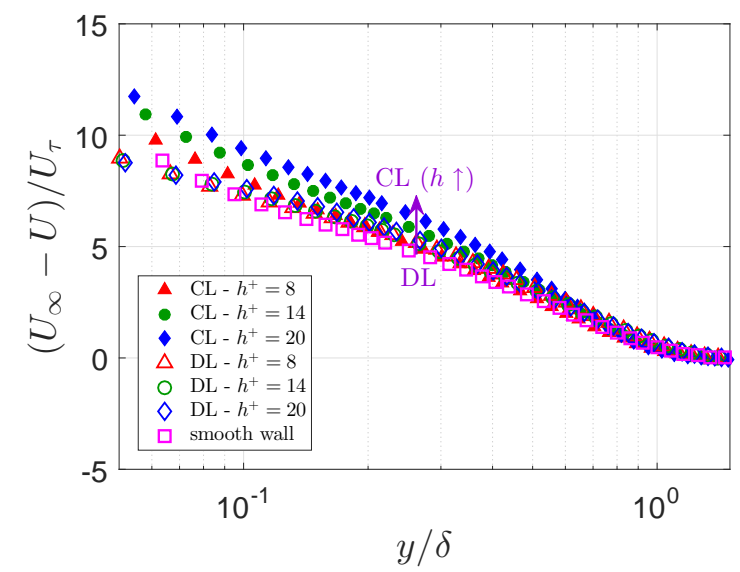

FIG. 11. Velocity decay profile $\left(U_{\infty}-U\right) / U_{\tau}$ as a function of outer coordinate $y / \delta$. The local friction velocity $U_{\tau}$ and boundary layer thickness $\delta$ are applied in the normalization.

which affects the velocity profile in the entire boundary layer. As the riblet height increases, the 460 velocity decay profile over the CL moves upward and hence deviates further from the smooth-wall case. Even with a tentative adjustment of the local friction velocity, an overlap of the velocity decay profiles in the outer region cannot be obtained. Nugroho et al. ${ }^{5}$ observed non-overlapping of velocity decay profiles over C-D riblets at a high yaw angle of $\gamma=30^{\circ}$. The friction velocity (wall-shear stress) may not be estimated directly using Eq. (6) by assuming Townsend's outer-layer 465 similarity. Furthermore, it was found that Townsend's outer-layer similarity hypothesis does not hold for other types of spanwise heterogeneous surface patterns, including spanwise alternating roughness strips ${ }^{61}$ or surface elevation. ${ }^{58}$ Thus, it is likely that the spanwise flow generated over a surface pattern with spanwise heterogeneity undermines the similarity in the outer layer.

In summary, C-D riblets lead to a significant change in the wall-shear stress, with an increase 470 over the CL and a decrease over the DL. The drag variations compared to the smooth-wall case become more pronounced as the riblet height increases. Townsend's outer-layer similarity hypothesis is not valid over C-D riblets in the present study.

\section{Population density of spanwise vortices}

The population distribution of spanwise vortices is obtained by the recognition and statistical 475 techniques described in Section III A. Figure 12 shows the population density of prograde vortices $\left(\Pi_{p}\right)$ and retrograde vortices $\left(\Pi_{r}\right)$. The population density of prograde vortices is larger than that of retrograde vortices, which can be attributed to the direction of the mean shear. In the tur- 
bulent boundary layer more prograde vortices which follow the direction of the mean shear are accounted. ${ }^{24,28}$ In general, an increased population density of prograde/retrograde vortices is ob480 served (except for the DL with $h^{+}=8$ ), with a more significant increase over the CL than the DL. The greater population of spanwise vortices over the CL was also qualitatively observed by Kevin et al. ${ }^{9}$ Over the CL, the population density of spanwise vortices in the boundary layer increases by more than $50 \%$ (more than two times in most of the regions for $h^{+}=20$ ) as compared with the smooth-wall case, implying a significant increase in the turbulence production activities. 485 Although the identified structures may be affected by the particular choice of structure and identification method, it is clear that the intensity of coherent motions over the converging region is significant increased. The significant increase over the CL confirms that there exists a creation of new coherent structures over the converging region, as proposed by Kevin et al. ${ }^{9}$ The change in the riblet height affects the whole boundary layer for prograde vortices and only the near-wall region 490 of $y / \delta_{s}<0.5$ for retrograde vortices, and the population density of the vortices presents a positive correlation with the riblet height. Over the CL, the vertical position with the maximum population density of retrograde vortices $\left(\Pi_{r}\right)$ shifts away from the wall as the riblet height increases, while a vertical shift of the peak for prograde vortices $\left(\Pi_{p}\right)$ is not apparent. The vertical shift of the $\Pi_{r}$ peak over the CL can be attributed to the more intense upwelling secondary flow, which are 495 essentially caused by the greater capacity of the spanwise flow with an increased riblet height. Over the DL, the population density of prograde and retrograde spanwise vortices remains almost unchanged in a large portion of the boundary layer except for some small changes in the near-wall area. We attribute these phenomena to the downwelling secondary flow, which transports the upper undisturbed fluid to the near-wall region. The population density of these spanwise vortices is 500 not sensitivity to an increase in the riblet height over the DL.

In summary, the population and distribution of prograde and retrograde spanwise vortices are compared to analyze the features of vortical structures over C-D riblets. The creation of new coherent structures can be confirmed by the significant modification of the population density of spanwise vortices over C-D riblets. Over the CL, the number of spanwise vortices is significantly 505 increased by more than $50 \%$ over even the lowest C-D riblets. Over the DL, the change of the population density of spanwise vortices is far less significant. A height increase of C-D riblets leads to a larger population of spanwise vortices over both the CL and the DL. Over the DL, a variation in the riblet height is primarily reflected in the near-wall region. 
(a)

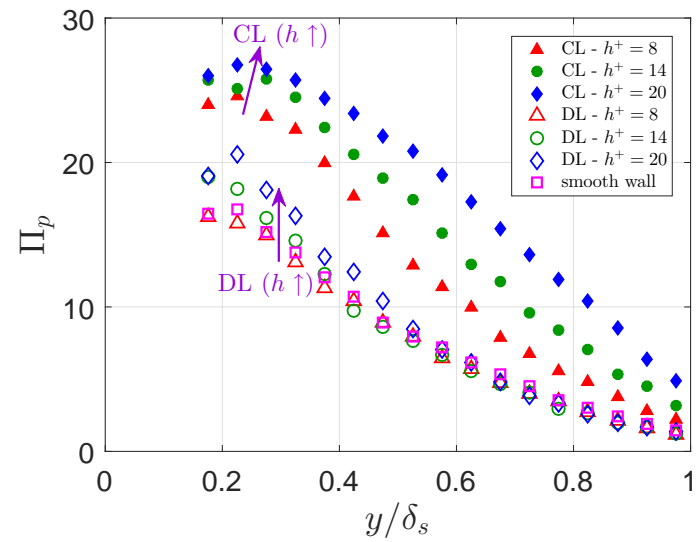

(b)

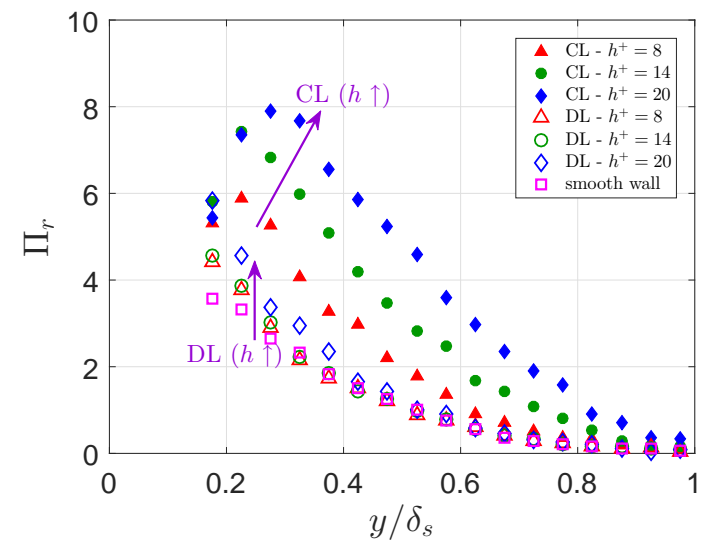

FIG. 12. Population density of $(a)$ prograde vortices $\Pi_{p},(b)$ retrograde vortices $\Pi_{r}$, as a function of outer coordinate $y / \delta_{s}$.

\section{Spatial correlation}

The features of vortex packets are obtained by the spatial correlation analysis in the longitudinal plane described in Section III B. Figure 13 shows six representative contours of $R_{u^{\prime} u^{\prime}}$ over the CL/DL of $h^{+}=14 \mathrm{C}$-D riblets and the smooth-wall case at two wall-normal stations of $y / \delta_{s}=0.2$ and $y / \delta_{s}=0.7$. Contour lines are between 0.3 and 0.8 with a spacing of 0.1 . The central dots indicate the correlation origins, the solid line indicates the streamwise length scale $L_{x}$, and the 515 dashed line shows the inclination angle $\alpha$. Only the case of $h^{+}=14$ is provided in Fig. 13, and the similar cases of $h^{+}=8$ and $h^{+}=20$ are not shown here for the sake of brevity. The boundary layer thickness over the smooth wall $\left(\delta_{s}\right)$ is applied to normalize the horizontal and vertical coordinates, which ensures a consistent physical station of correlation origins. If the local boundary layer thickness was applied to normalization, a comparison of the length scales would be misleading.

These contours indicate a distinct structure which is inclined at a shallow angle in the streamwise direction. ${ }^{32}$ For the correlation origin at $y / \delta_{s}=0.2$, the contour over the C-D riblets extends to a shorter streamwise distance in comparison with that over the smooth wall, and inclines at a steeper (shallower) angle over the CL (DL), as indicated in Fig. 13c (Fig. 13e). The contour shape with the correlation origin at $y / \delta_{s}=0.7$ is indicative of the spatial arrangement of vortex packets found in 525 the outer region. ${ }^{36,62}$ The differences in the contour shapes over the CL in Fig. 13d and the smooth wall in Fig. 13b are not significant. Over the DL, the contour in Fig. 13f extends to a shorter streamwise distance and inclines at a steeper angle.

To quantitatively extract the features of vortex packets, the preceding contours are analyzed in 
(a)

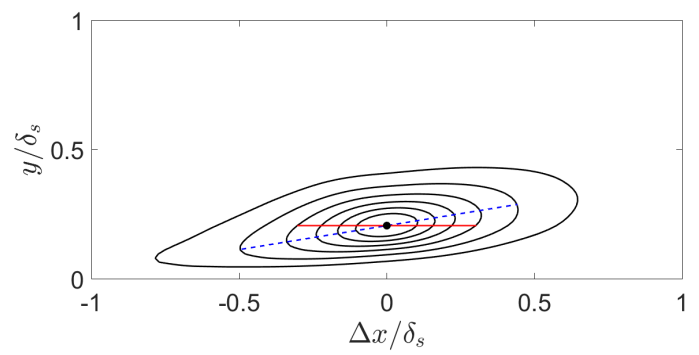

(c)

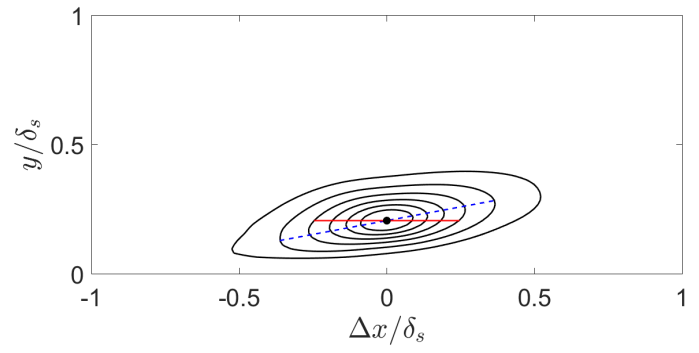

(e)

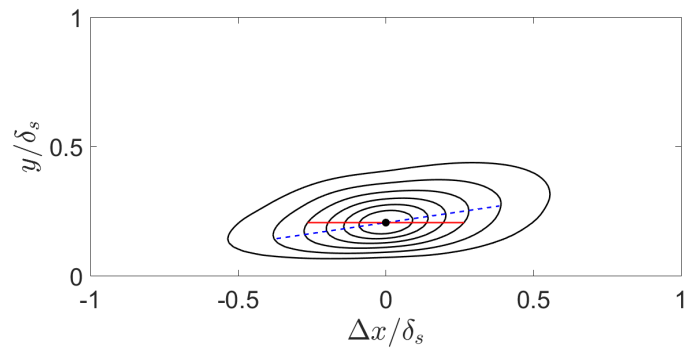

(b)

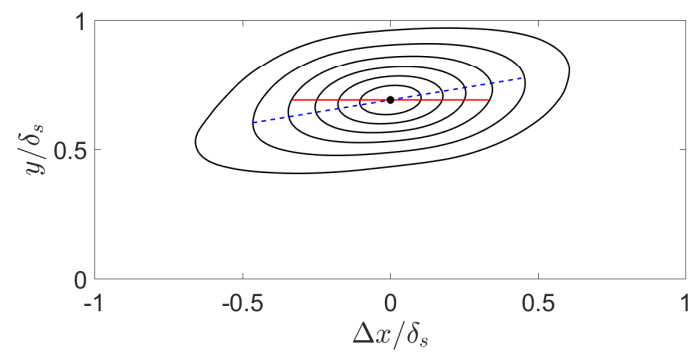

(d)

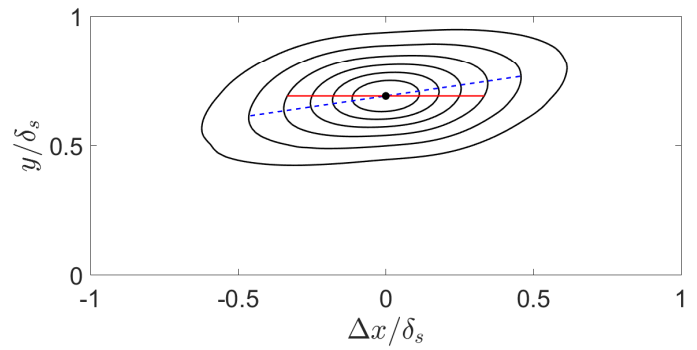

(f)

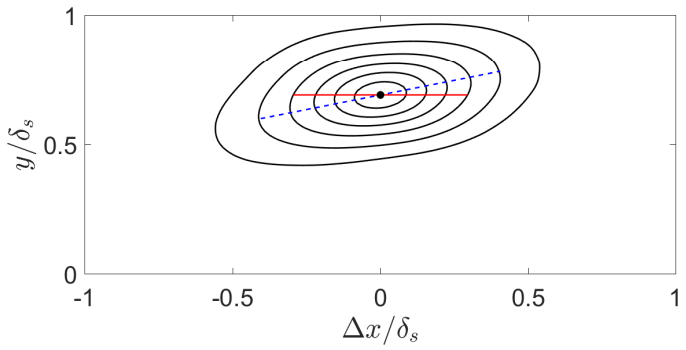

FIG. 13. Correlation contours of the streamwise fluctuating velocity in the longitudinal plane along $(a)$ smooth wall at $y / \delta_{s}=0.2,(b)$ smooth wall at $y / \delta_{s}=0.7,(c) h^{+}=14-\mathrm{CL}$ at $y / \delta_{s}=0.2,(d) h^{+}=14-\mathrm{CL}$ at $y / \delta_{s}=0.7,(e) h^{+}=14-\mathrm{DL}$ at $y / \delta_{s}=0.2,(f) h^{+}=14-\mathrm{DL}$ at $y / \delta_{s}=0.7$.

terms of the streamwise length and the inclination angle. Figure 14a compares the streamwise 530 length scale $\left(L_{x}\right)$ extracted at the contour level of $R_{u^{\prime} u^{\prime}}=0.5$. Over the smooth wall, $L_{x}$ decreases rapidly in the region of $y / \delta_{s}<0.2$, and then presents a slight increase followed by a mild decrease, with an approximate platform at $L_{x} \approx 0.65 \delta_{s}$ in the region of $0.4<y / \delta_{s}<0.8$. Near the edge of the boundary layer, $L_{x}$ decreases rapidly as a consequence of the low freestream turbulence. Both the changing trend and the platform value of $L_{x}$, including the rapid drop in the near-wall region, 535 agree well with the result by Volino et al. ${ }^{33}$ For C-D riblets, over the CL, $L_{x}$ is significantly smaller in $y / \delta_{s}<0.5$ and slight larger in $y / \delta_{s}>0.65$ than that over the smooth wall, and the platform area does not exist. A lower (higher) value of $L_{x}$ indicates a reduced (increased) streamwise spacing between consecutive vortices in the vortex packets. ${ }^{63}$ The smaller $L_{x}$ in the region of $y / \delta_{s}<0.5$ over the CL can be attributed to the larger population of spanwise vortices as revealed in Fig. 12, 540 i.e., the creation of new coherent structures. Similarly, over the rough wall made of a woven mesh surface, Volino et al. ${ }^{33}$ also observed a lower $L_{x}$ in the near-wall region compared with the 
smooth wall. However, the mechanism of the rough wall is attributed to the destruction of the near-wall streaks, since no upwelling secondary flow occurs in the time-averaged sense. Due to the upwelling over the converging region, the area with a lower value of $L_{x}$ over the CL of C-D 545 riblets $\left(y / \delta_{s}<0.5\right)$ is much larger than that of the rough wall $\left(y / \delta_{s}<0.1\right)$. The riblet height has little effect on $L_{x}$ over the CL, with an increased riblet height leading to a slightly larger value of the streamwise length in the outer region. Over the DL, a narrower streamwise length $L_{x}$ of the $R_{u^{\prime} u^{\prime}}$ contour than that over the smooth wall is observed in the entire boundary layer, with an increased riblet height leading to a shorter streamwise length of vortex packets. The platform region of $L_{x}$ 550 exists over the DL, but does not present over the CL. A change in the riblet height corresponds to a significant variation of $L_{x}$ over the DL. Volino et al. ${ }^{33}$ showed that a homogeneous rough surface only modifies $L_{x}$ in the near-wall area, whereas the feature of $L_{x}$ in the outer flow remains essentially unchanged. In contrast, the C-D riblets profoundly modify the spatial distribution of coherent structures by introducing a large-scale secondary flow, leading to a significant change in $555 L_{x}$ across the entire boundary layer.

Figure 14b shows the wall-normal variation of the inclination angle $(\alpha)$ over the C-D riblets and the smooth wall. Over the smooth wall, the inclination angle is approximately $10.5^{\circ}$ for $0.2<y / \delta_{s}<0.7$ in the present study, which is close to the results obtained in other studies (e.g., $10^{\circ}$ by Krogstad \& Antonia, ${ }^{64} 12^{\circ}$ by Adrian et al., ${ }^{36} 12^{\circ}-13^{\circ}$ by Christensen $\&$ Adrian, ${ }^{62} 11^{\circ}$ 560 by Christensen $\& \mathrm{Wu},{ }^{32} 10.7^{\circ}-15.7^{\circ}$ by Volino et al. $\left.{ }^{33}\right)$. Compared with the smooth wall, the inclination angle is larger (smaller) over the CL (DL) in the near-wall region of $y / \delta_{s}<0.25$. The larger (smaller) inclination angle over the CL (DL) can be tentatively attributed to the upwelling (downwelling) in the near-wall region. Similarly, a larger inclination is observed over the upwelling (elevated) region over an elevation heterogeneity, which is attributed to the enhanced 565 mixing within the high momentum pathways. ${ }^{65}$ Away from the wall, the inclination angle in the region of $y / \delta_{s}>0.25$ is larger over the DL and slightly smaller over the CL compared with the smooth wall. The steeper hairpin packet in the outer region over the DL may be attributed to the smaller number of spanwise vortices, i.e., fewer hairpin vortices in the boundary layer. The riblet height has a negligible effect on the inclination angle in the region between $0.3 \delta_{s}$ and $0.7 \delta_{s}$. The 570 rough wall is observed to lead to an inclination angle $1^{\circ}$ to $3^{\circ}$ larger than that over the smoothwall case. ${ }^{33,66}$ For the C-D riblets, the changing trend of the inclination angle is inconsistent in the boundary layer, e.g., an increased $\alpha$ occurs only in the near-wall region over the CL and only in the outer region over the DL. 
(a)

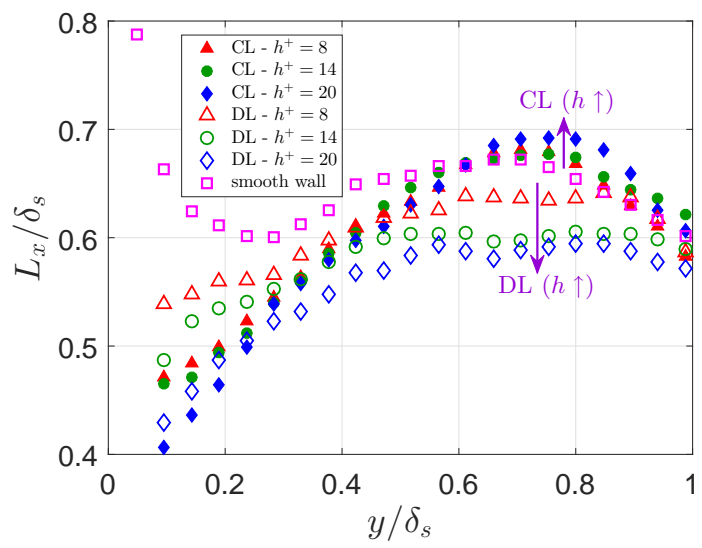

(b)

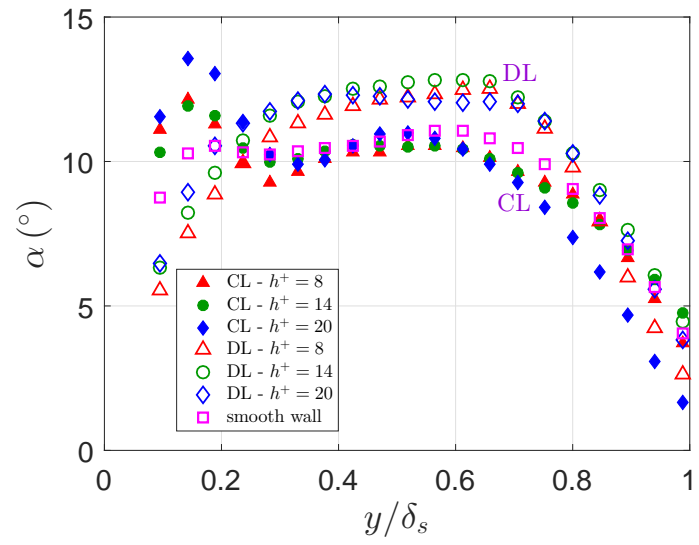

FIG. 14. Profiles of $(a)$ streamwise length scale $L_{x},(b)$ inclination angle $\alpha$, of the streamwise velocity correlation contour as a function of outer coordinate $y / \delta_{s}$.

(a) smooth

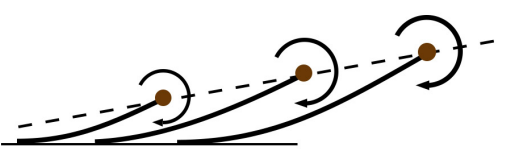

(b) $\mathrm{CL}$

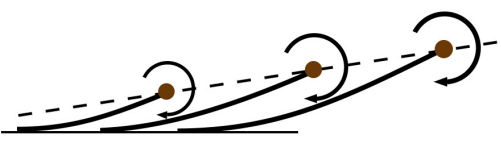

(c) $\mathrm{DL}$

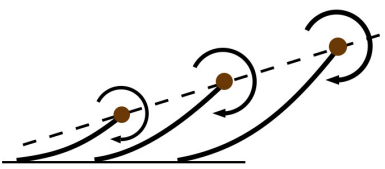

FIG. 15. Schematic diagram of vortex packet in the outer region over $(a)$ smooth wall, $(b)$ CL of C-D riblets, (c) DL of C-D riblets, indicating its streamwise length scale and inclination angle. The brown dots indicate the heads of hairpin vortices.

In summary, the spatial correlation characteristics in the longitudinal plane suggest that C-D 575 riblets lead to a significantly modified spatial arrangement of vortex packets. By synthesizing Figs. 14a and 14b, schematics of the shapes of vortex packets in the outer region are shown in Fig. 15. In comparison with the smooth-wall case, while the $\delta$-scale vortex packets are steeper and less stretched in the outer region over the diverging region, they are less affected (slightly flatter and a bit more stretched) over the converging region. An increase in the riblet height results in a 580 further shortening of these packets in the streamwise direction over the diverging region.

\section{E. Uniform momentum zones}

The uniform momentum zones (UMZs) are identified by the technique described in Section III C. Figure 16a compares the p.d.f. of the number of UMZs $\left(N_{U M Z}\right)$ over the C-D riblets and the smooth wall. The dominant number of UMZs, i.e., $N_{U M Z}$ with the highest probability, changes 
(a)

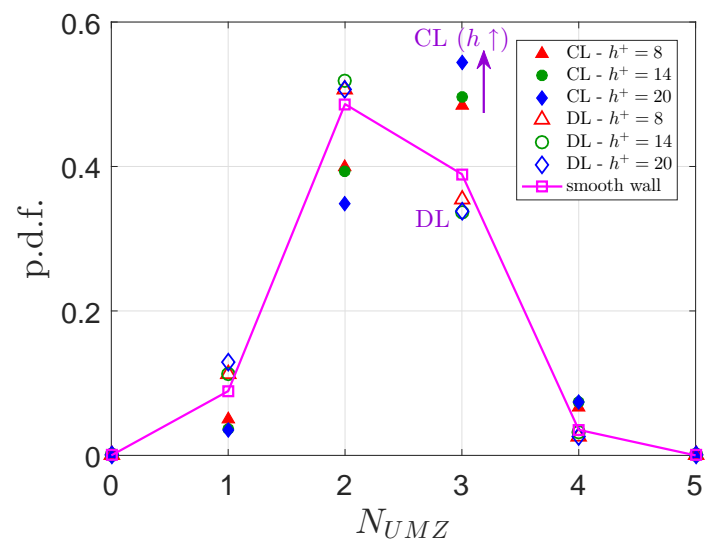

(b)

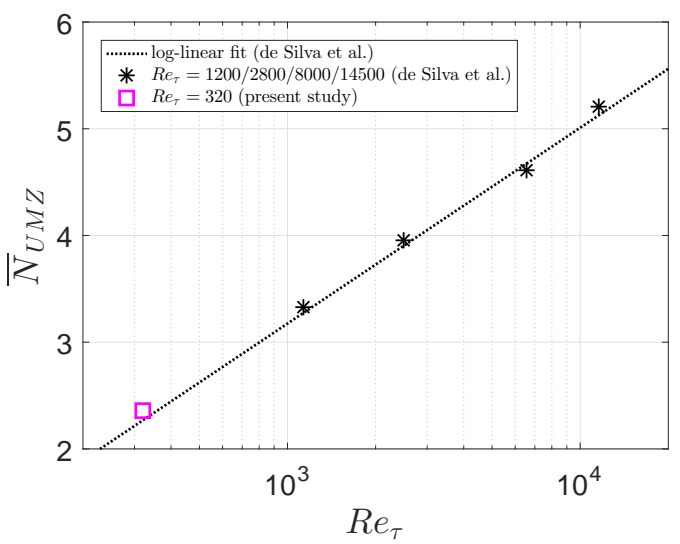

FIG. 16. (a) P.d.f. of the number of UMZs $N_{U M Z}$. (b) Mean number of UMZs $\bar{N}_{U M Z}$ as a function of friction Reynolds number $R e_{\tau}$.

585 from $N_{U M Z}=2$ over the smooth wall or the DL to $N_{U M Z}=3$ over the CL. The average number of UMZs $\left(\bar{N}_{U M Z}\right)$ can be calculated by the probability weighted summation of $N_{U M Z}$ in Fig. 16a. As stated by de Silva et al. ${ }^{34} \bar{N}_{U M Z}$ presents a log-linear increase with increasing friction Reynolds number $\left(R e_{\tau}\right)$ at medium to high Reynolds numbers. The value of $\bar{N}_{U M Z}$ over the smooth wall at the present Reynolds number is 2.371 , which also follows this log-linear relationship as indicated 590 in Fig. 16b. The consistency here provides further support to the hierarchical length scale distribution of vortical structures within the boundary layer. ${ }^{67} \bar{N}_{U M Z}$ is larger over the CL than the smooth wall, and the riblet height has a positive correlation with $\bar{N}_{U M Z}\left(\bar{N}_{U M Z}=2.567 / 2.608 / 2.658\right.$ for C-D riblets with $h^{+}=8 / 14 / 20$ respectively). Over the CL, an increased riblet height leads to a more intense common-flow upwelling and a higher population density of hairpin vortices, which 595 directly contribute to more UMZs. A similarity exists over the CL between the $h^{+}=8$ and $h^{+}=14$ cases, followed by a large difference to the $h^{+}=20$ case. On the contrary, $\bar{N}_{U M Z}$ is lower over the DL in comparison with the smooth wall $\left(\bar{N}_{U M Z}=2.295 / 2.290 / 2.260\right.$ for C-D riblets with $h^{+}=8 / 14 / 20$ respectively), while the riblet height has a negligible effect on the distribution of $N_{U M Z}$. The insensitivity of $\bar{N}_{U M Z}$ to the riblet height over the DL may be attributed to the 600 time-averaged downwelling. The downwelling secondary flow, which transports the fluid from the upper region to the riblet surface, inhibits its direct influence on the spatial distribution of the upper vortical structures. ${ }^{7}$

Figure 17a compares the p.d.f. of the mean thickness of UMZs $\left(\bar{t}_{U M Z}\right)$ over the C-D riblets and the smooth wall. The calculation of the UMZ thickness at a certain streamwise station is ${ }_{605}$ indicated in Fig. 8b, and its averaged value in the streamwise direction is defined as the mean 
(a)

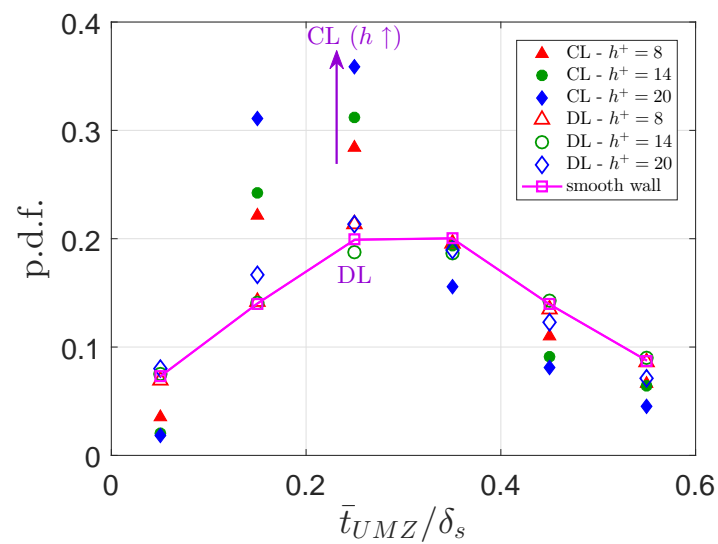

(b)

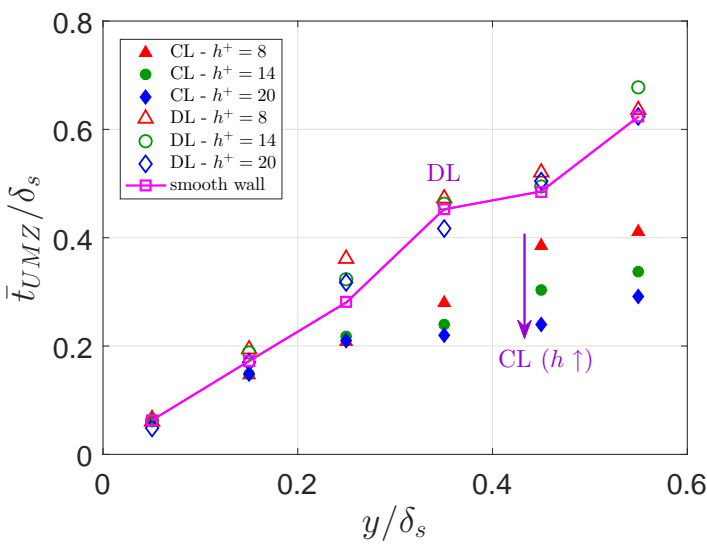

FIG. 17. (a) P.d.f. of the mean thickness of UMZs $\bar{t}_{U M Z}$ normalized by $\delta_{s}$. (b) Mean thickness of UMZs $\bar{t}_{U M Z}$ as a function of outer coordinate $y / \delta_{s}$.

UMZ thickness. Over the CL, the p.d.f. of the UMZ with a thickness of $\bar{t}_{U M Z} / \delta_{s} \approx 0.2$ increases dramatically. An increase in the riblet height leads to a higher probability of $\bar{t}_{U M Z} / \delta_{s} \approx 0.2$. The significant change over the CL can be primarily attributed to the rearrangement of the newly created coherent structures over the converging region. ${ }^{9}$ The p.d.f. of the UMZ thickness over the DL remains basically unchanged compared with that over the smooth wall.

Figure 17b shows the wall-normal variation of the mean thickness of UMZs $\left(\bar{t}_{U M Z}\right)$ over C-D riblets and the smooth wall. The wall-normal station of a UMZ in a snapshot is represented by the $y$ coordinate of its geometric center. For all cases, the normalized UMZ thickness $\left(\bar{t}_{U M Z} / \delta_{s}\right)$ increases as its wall-normal station moves away from the wall. Over the CL, the mean UMZ 615 thickness is significantly reduced in the region of $y / \delta_{s}>0.2$, while in the near-wall region of $y / \delta_{s}<0.2$ the difference from the smooth-wall case is negligible. The slope of the curves over the CL is significantly lower than that over the smooth wall or the DL. The reduced mean UMZ thickness over the CL in the region of $0.2<y / \delta_{s}<0.4$ helps explain the significantly increased probability of $\bar{t}_{U M Z} / \delta_{s} \approx 0.2$ as indicated in Fig. 17a. An increase in the riblet height leads to a 620 more significant decrease in the thickness of UMZs in the region away from the wall over the CL. As validated in Fig. 12, due to the creation of new coherent structures, the population density of spanwise vortices increases significantly over the CL. The reduced $\bar{t}_{U M Z} / \delta_{s}$ over the CL is directly related to the reorganization of these coherent structures. Over the DL, C-D riblets lead to a slight increase in the mean thickness of UMZs compared with the smooth-wall case, which is aligned ${ }_{625}$ with the slightly decreased value of $\bar{N}_{U M Z}$.

In summary, for the uniform momentum zones, C-D riblets result in a larger ensemble-averaged 
number over the CL and a slightly smaller number over the DL, compared with the smooth-wall case. The thickness of UMZs increases as the station moves away from the wall. Compared with the smooth-wall case, UMZs are thinner over CL especially in the area far above the wall, which is 630 attributed to the upwelling and the spatial rearrangement of the newly created coherent structures. A greater riblet height results in a more profound modification to the UMZ features over the CL. Over the DL, the effect of C-D riblet on the thickness of UMZs is not significant.

\section{CONCLUSIONS}

A particle image velocimetry (PIV) study of the turbulent boundary layers developing over 635 convergent-divergent riblets (C-D riblets) of three different heights $\left(h^{+}=8,14\right.$ and 20) is undertaken at $R e_{\theta}=723$. The purposes of this study are firstly to examine the effect of C-D riblets on the behaviors of turbulent structures in comparison to the smooth-wall case and the differences in such behaviors between the converging region and the diverging region in a more quantitative manner, and secondly to establish a better understanding of how riblet height affects such behaviors.

It is found that in comparison to the baseline case, an increase in the time-averaged streamwise velocity and a reduction in the time-averaged Reynolds shear stress are observed in the near-wall region over the diverging line. In contrast, the opposite occurs over the converging line and the impact extends across almost the entire boundary layer. An increase in riblet height enhances such a trend and affects the profiles of velocity and Reynolds shear stress more profoundly over 645 the converging line. Furthermore, it is observed that although a logarithmic law region is present in the velocity profiles over the converging and diverging line, Townsend's outer-layer similarity hypothesis is invalid.

With a large sample size of PIV snapshots available, the population of prograde and retrograde spanwise vortices are investigated. It is found that with a riblet height of $h^{+}=8(2.4 \%$ of the 650 baseline boundary layer thickness), the population densities of prograde and retrograde spanwise vortices are increased by more than $50 \%$ over the converging region compared to the smoothwall case, implying a significant increase in the turbulence production activities. An increase in the height of C-D riblets results in a substantial increase in the population of both prograde and retrograde spanwise vortices. Over the diverging region, except for the small changes in the near655 wall region, the population density of prograde and retrograde spanwise vortices remains almost unaffected in a large portion of the boundary layer, and it appears to be much less sensitive to an 
increase in the riblet height.

The inclination angle and streamwise extent of vortex packets are also extracted from twopoint correlations. In comparison with the smooth-wall case, while the $\delta$-scale vortex packets are ${ }_{660}$ steeper and less stretched in the outer region over the diverging region, they are less affected over the converging region. The effect of an increasing riblet height is mainly manifested as a further shortening of these packets in the streamwise direction over the diverging region.

Finally, the zonal behavior of uniform streamwise momentum in the boundary layer is examined. It is observed that, in comparison with the smooth-wall case, over the converging region 665 while the probability of having three or four uniform momentum zones increases, the probability of having one or two uniform momentum zones decreases at the Reynolds number investigated in the present study. Correspondingly, the uniform momentum zones in the outer part of the boundary layer over the converging region become thinner. Again, an increase in the riblet height enhances such a trend.

Overall, it is observed that while an increase in the riblet height affects a large portion of the boundary layer away from the wall over the converging region, the impact on the diverging region is largely confined within the near-wall region. Such distinct differences in the response of the boundary layer over the diverging and converging region is attributed to the opposite local secondary flow motion induced by the riblets.

\section{ACKNOWLEDGMENTS}

The first author wishes to acknowledge the President's Doctoral Scholar award from the University of Manchester. The authors would like to thank the technical supports from workshop technicians at the School of Mechanical, Aerospace and Civil Engineering. Finally, the authors would like to thank the referees of this paper for their valuable review comments.

\section{REFERENCES}

${ }^{1}$ H. Chen, F. Rao, X. Shang, D. Zhang, and I. Hagiwara, "Flow over bio-inspired 3D herringbone wall riblets," Exp. Fluids 55, 1698 (2014).

${ }^{2}$ Q. Liu, S. Zhong, and L. Li, "Reduction of pressure losses in a linear cascade using herring- 
bone riblets," in Proc. ASME Turbo Expo 2017 Turbomach. Tech. Conf. Expo. (Charlotte, North Carolina, USA, 2017).

${ }^{3}$ P. Quan, S. Zhong, Q. Liu, and L. Li, "Attenuation of flow separation using herringbone riblets at $M_{\infty}=5, "$ AIAA J. 57, 142-152 (2019).

${ }^{4}$ K. Koeltzsch, A. Dinkelacker, and R. Grundmann, "Flow over convergent and divergent wall riblets," Exp. Fluids 33, 346-350 (2002).

${ }^{5}$ B. Nugroho, N. Hutchins, and J. P. Monty, "Large-scale spanwise periodicity in a turbulent boundary layer induced by highly ordered and directional surface roughness," Int. J. Heat Fluid Flow 41, 90-102 (2013).

${ }^{6}$ Kevin, J. P. Monty, H. L. Bai, G. Pathikonda, B. Nugroho, J. M. Barros, K. T. Christensen, and N. Hutchins, "Cross-stream stereoscopic particle image velocimetry of a modified turbulent boundary layer over directional surface pattern,” J. Fluid Mech. 813, 412-435 (2017).

${ }^{7} \mathrm{~F}$. Xu, S. Zhong, and S. Zhang, "Vortical structures and development of laminar flow over convergent-divergent riblets," Phys. Fluids 30, 051901 (2018).

${ }^{8}$ F. Xu, S. Zhong, and S. Zhang, "Vortical structures of laminar boundary layer over convergentdivergent riblets with spanwise height variations," in 21st Australas. Fluid Mech. Conf. (Adelaide, Australia, 2018).

${ }^{9}$ Kevin, J. Monty, and N. Hutchins, “Turbulent structures in a statistically three-dimensional boundary layer,” J. Fluid Mech. 859, 543-565 (2019).

${ }^{10}$ D. Willingham, W. Anderson, K. T. Christensen, and J. M. Barros, "Turbulent boundary layer flow over transverse aerodynamic roughness transitions: Induced mixing and flow characterization,” Phys. Fluids 26, 025111 (2014).

${ }^{11}$ H. L. Bai, Kevin, N. Hutchins, and J. P. Monty, "Turbulence modifications in a turbulent boundary layer over a rough wall with spanwise-alternating roughness strips,” Phys. Fluids 30, 055105 (2018)

${ }^{12}$ I. Nezu and H. Nakagawa, "Cellular secondary currents in straight conduit," J. Hydraul. Eng. 110, 173-193 (1984).

${ }^{13} \mathrm{~J}$. Yang and W. Anderson, "Numerical study of turbulent channel flow over surfaces with variable spanwise heterogeneities: topographically-driven secondary flows affect outer-layer similarity of turbulent length scales," Flow, Turbul. Combust. 100, 1-17 (2018).

${ }^{14}$ H. G. Hwang and J. H. Lee, "Secondary flows in turbulent boundary layers over longitudinal surface roughness,” Phys. Rev. Fluids 3, 014608 (2018). 
${ }^{15}$ L. Djenidi, F. Anselmet, J. Liandrat, and L. Fulachier, "Laminar boundary layer over riblets," Phys. Fluids 6, 2993-2999 (1994).

${ }^{16}$ G. R. Grek, V. V. Kozlov, S. V. Titarenko, and B. G. B. Klingmann, "The influence of riblets on a boundary layer with embedded streamwise vortices," Phys. Fluids 7, 2504-2506 (1995).

${ }_{720}{ }^{17} \mathrm{~S}$. Raayai-Ardakani and G. H. McKinley, "Drag reduction using wrinkled surfaces in high Reynolds number laminar boundary layer flows," Phys. Fluids 29, 093605 (2017).

${ }^{18}$ N. Yadav, S. W. Gepner, and J. Szumbarski, "Instability in a channel with grooves parallel to the flow," Phys. Fluids 29, 084104 (2017).

${ }^{19}$ H. Q. Ho and M. Asai, "Experimental study on the stability of laminar flow in a channel with 725 streamwise and oblique riblets," Phys. Fluids 30, 024106 (2018).

${ }^{20}$ J. H. Ng, R. K. Jaiman, and T. T. Lim, "Interaction dynamics of longitudinal corrugations in Taylor-Couette flows," Phys. Fluids 30, 093601 (2018).

${ }^{21}$ N. Yadav, S. W. Gepner, and J. Szumbarski, "Flow dynamics in longitudinally grooved duct," Phys. Fluids 30, 104105 (2018).

${ }_{730}{ }^{22}$ B. Nugroho, Kevin, J. Monty, N. Hutchins, and E. Gnanamanickam, "Roll-modes generated in turbulent boundary layers with passive surface modifications," in 52nd Aerosp. Sci. Meet. (National Harbor, Maryland, 2014) pp. AIAA 2014-0197.

${ }^{23}$ Kevin, B. Nugroho, J. P. Monty, and N. Hutchins, "Wall-parallel PIV measurements in turbulent boundary layers with highly directional surface roughness," in 19th Australas. Fluid Mech. Conf.

735 (Melbourne, Australia, 2014).

${ }^{24}$ R. J. Adrian, "Hairpin vortex organization in wall turbulence,” Phys. Fluids 19, 041301 (2007).

${ }^{25} \mathrm{X}$. Wu and P. Moin, "Forest of hairpins in a low-Reynolds-number zero-pressure-gradient flatplate boundary layer," Phys. Fluids 21, 091106 (2009).

${ }^{26}$ G. Eitel-Amor, R. Örlü, P. Schlatter, and O. Flores, "Hairpin vortices in turbulent boundary 740 layers," Phys. Fluids 27, 025108 (2015).

${ }^{27}$ H. Tian, J. Zhang, N. Jiang, and Z. Yao, "Effect of hierarchical structured superhydrophobic surfaces on coherent structures in turbulent channel flow," Exp. Therm. Fluid Sci. 69, 27-37 (2015).

${ }^{28}$ Y. Wu and K. T. Christensen, "Population trends of spanwise vortices in wall turbulence," J. 745 Fluid Mech. 568, 55-76 (2006).

${ }^{29}$ V. K. Natrajan, Y. Wu, and K. T. Christensen, "Spatial signatures of retrograde spanwise vortices in wall turbulence,” J. Fluid Mech. 574, 155-167 (2007). 
${ }^{30}$ Y. Hwang and C. Cossu, "Self-sustained process at large scales in turbulent channel flow," Phys. Rev. Lett. 105, 044505 (2010).

${ }^{750}{ }^{31}$ S. Rawat, C. Cossu, Y. Hwang, and F. Rincon, "On the self-sustained nature of large-scale motions in turbulent Couette flow," J. Fluid Mech. 782, 515-540 (2015).

${ }^{32} \mathrm{~K}$. Christensen and $\mathrm{Y}$. Wu, "Characteristics of vortex organization in the outer layer of wall turbulence," in Fourth Int. Symp. Turbul. Shear Flow Phenom. (Begel House Inc., 2005) pp. 1025-1030.

${ }_{755}^{33}$ R. J. Volino, M. P. Schultz, and K. A. Flack, "Turbulence structure in rough- and smooth-wall boundary layers," J. Fluid Mech. 592, 263-293 (2007).

${ }^{34}$ C. M. de Silva, N. Hutchins, and I. Marusic, "Uniform momentum zones in turbulent boundary layers,” J. Fluid Mech. 786, 309-331 (2016).

${ }^{35}$ C. D. Meinhart and R. J. Adrian, "On the existence of uniform momentum zones in a turbulent 760 boundary layer," Phys. Fluids 7, 694-696 (1995).

${ }^{36}$ R. J. Adrian, C. D. Meinhart, and C. D. Tomkins, "Vortex organization in the outer region of the turbulent boundary layer," J. Fluid Mech. 422, 1-54 (2000).

${ }^{37}$ Y. Wu and K. T. Christensen, "Outer-layer similarity in the presence of a practical rough-wall topography," Phys. Fluids 19, 085108 (2007).

${ }_{765}^{38}$ K. A. Flack, M. P. Schultz, and J. S. Connelly, "Examination of a critical roughness height for outer layer similarity," Phys. Fluids 19, 095104 (2007).

${ }^{39}$ R. García-Mayoral and J. Jiménez, "Drag reduction by riblets,” Philos. Trans. R. Soc. A Math. Phys. Eng. Sci. 369, 1412-1427 (2011).

${ }^{40}$ F. H. Clauser, “Turbulent boundary layers in adverse pressure gradients," J. Aeronaut. Sci. 21, $770 \quad 91-108$ (1954).

${ }^{41}$ R. Vinuesa and R. Örlü, "Measurement of wall-shear stress," in Exp. Aerodyn., edited by S. Discetti and A. Ianiro (CRC Press, 2017) pp. 393-428.

${ }^{42}$ L. P. Erm, P. N. Joubert, and A. J. Smits, “Low Reynolds number turbulent boundary layers on a smooth flat surface in a zero pressure gradient," in Turbul. Shear Flows 5, edited by F. Durst, 775 B. E. Launder, J. L. Lumley, F. W. Schmidt, and J. H. Whitelaw (Springer, Cornell University, Ithaca, New York, USA, 1985) pp. 186-196.

${ }^{43}$ L. P. Erm and P. N. Joubert, "Low-Reynolds-number turbulent boundary layers," J. Fluid Mech. 230, 1-44 (1991).

${ }^{44}$ P. R. Spalart, "Direct simulation of a turbulent boundary layer up to $R e_{\theta}=1410$," J. Fluid Mech. 
${ }^{45} \mathrm{X}$. Wu and P. Moin, "Direct numerical simulation of turbulence in a nominally zero-pressuregradient flat-plate boundary layer," J. Fluid Mech. 630, 5-41 (2009).

${ }^{46}$ J. D. Cameron, S. C. Morris, S. Bailey, and A. J. Smits, "Effects of hot-wire length on the measurement of turbulent spectra in anisotropic flows," Meas. Sci. Technol. 21, 105407 (2010).

${ }_{785}{ }^{47}$ J. Zhou, R. J. Adrian, S. Balachandar, and T. M. Kendall, "Mechanisms for generating coherent packets of hairpin vortices in channel flow,” J. Fluid Mech. 387, 353-396 (1999).

${ }^{48}$ T. H. Cormen, C. E. Leiserson, R. L. Rivest, and C. Stein, Introduction to algorithms, 3rd ed. (The MIT Press, 2009).

${ }^{49}$ L. Li, S. Erfani, and C. Leckie, "A pattern tree based method for mining conditional contrast 790 patterns of multi-source data," in 2017 IEEE Int. Conf. Data Min. Work. (IEEE, New Orleans, LA, 2017) pp. 916-923.

${ }^{50}$ M. Atzori, R. Vinuesa, A. Lozano-Durán, and P. Schlatter, "Characterization of turbulent coherent structures in square duct flow,” J. Phys. Conf. Ser. 1001, 012008 (2018).

${ }^{51}$ O. J. H. Williams, D. Sahoo, M. L. Baumgartner, and A. J. Smits, "Experiments on the structure 795 and scaling of hypersonic turbulent boundary layers,” J. Fluid Mech. 834, 237-270 (2018).

${ }^{52}$ I. Marusic and W. D. C. Heuer, "Reynolds number invariance of the structure inclination angle in wall turbulence," Phys. Rev. Lett. 99, 114504 (2007).

${ }^{53}$ K. C. Kim and R. J. Adrian, "Very large-scale motion in the outer layer,” Phys. Fluids 11, 417422 (1999).

${ }_{800}{ }^{54}$ C. M. de Silva, J. Philip, K. Chauhan, C. Meneveau, and I. Marusic, "Multiscale geometry and scaling of the turbulent-nonturbulent interface in high Reynolds number boundary layers," Phys. Rev. Lett. 111, 044501 (2013).

${ }^{55}$ F. Xu, Z. Gao, X. Ming, L. Xia, Y. Wang, W. Sun, and R. Ma, "The optimization for the backward-facing step flow control with synthetic jet based on experiment," Exp. Therm. Fluid 805 Sci. 64, 94-107 (2015).

${ }^{56}$ A. Vidal, H. M. Nagib, P. Schlatter, and R. Vinuesa, "Secondary flow in spanwise-periodic in-phase sinusoidal channels," J. Fluid Mech. 851, 288-316 (2018).

${ }^{57}$ A. E. Perry and J. D. Li, "Experimental support for the attached-eddy hypothesis in zeropressure-gradient turbulent boundary layers,” J. Fluid Mech. 218, 405 (1990).

${ }_{810}{ }^{58} \mathrm{~T}$. Medjnoun, C. Vanderwel, and B. Ganapathisubramani, "Characteristics of turbulent boundary layers over smooth surfaces with spanwise heterogeneities,” J. Fluid Mech. 838, 516-543 (2018). 
${ }^{59}$ P. Luchini, F. Manzo, and A. Pozzi, "Resistance of a grooved surface to parallel flow and crossflow,' J. Fluid Mech. 228, 87-109 (1991).

${ }^{60}$ K. Kamrin, M. Z. Bazant, and H. A. Stone, "Effective slip boundary conditions for arbitrary 815 periodic surfaces: the surface mobility tensor,” J. Fluid Mech. 658, 409-437 (2010).

${ }^{61}$ D. Chung, J. P. Monty, and N. Hutchins, "Similarity and structure of wall turbulence with lateral wall shear stress variations," J. Fluid Mech. 847, 591-613 (2018).

${ }^{62}$ K. T. Christensen and R. J. Adrian, "Statistical evidence of hairpin vortex packets in wall turbulence," J. Fluid Mech. 431, 433-443 (2001).

${ }_{820}^{63} \mathrm{Y}$. Wu and K. T. Christensen, "Spatial structure of a turbulent boundary layer with irregular surface roughness,” J. Fluid Mech. 655, 380-418 (2010).

${ }^{64}$ P. A. Krogstad and R. A. Antonia, "Structure of turbulent boundary layers on smooth and rough walls,” J. Fluid Mech. 277, 1-21 (1994).

${ }^{65} \mathrm{~A}$. Awasthi and W. Anderson, "Numerical study of turbulent channel flow perturbed by span825 wise topographic heterogeneity: Amplitude and frequency modulation within low- and highmomentum pathways," Phys. Rev. Fluids 3, 044602 (2018).

${ }^{66} \mathrm{~S}$. Nakagawa and T. J. Hanratty, "Particle image velocimetry measurements of flow over a wavy wall," Phys. Fluids 13, 3504-3507 (2001).

${ }^{67}$ I. Marusic, “On the role of large-scale structures in wall turbulence,” Phys. Fluids 13, 735-743 $830 \quad$ (2001). 